COMMUNICATIONS IN

ANALYSIS AND GEOMETRY

Volume 5, Number 3, 375-412, 1997

\title{
Heegaard structures of negatively curved 3-manifolds
}

\author{
YOAV MORIAH $^{1}$ AND HyAm RUBINSTEIN ${ }^{2}$
}

\section{Introduction.}

Let $M$ be an orientable complete hyperbolic 3-manifold of finite volume and with $d$ ordered cusps $c_{1}, \ldots, c_{d}$. Denote by $\mathbb{M}$ the collection of all manifolds obtained by Dehn surgery on $M$ i.e., if $q \in \mathbb{Q}^{d}, q=\left(q_{1}, \ldots, q_{d}\right)$, let $M_{q}$ denote the manifold obtained by $q_{i}$ surgery on the $i$-th cusp, $i=1, \ldots, d$, with respect to some prechosen basis for the cuspital homology. Note that topologically $M$ is contained in $M_{q}$ for all $q$. By a Heegaard splitting for $M$ we mean a decomposition of $M$ into two compression bodies as in [CG].

Thurston and Jorgenson (see [Th1]) proved that the collection of complete hyperbolic manifolds with finite volume is a well ordered set. The limit points are the manifolds $M$ with cusps and the manifolds $M_{q}$ converge to $M$ in the sense of Gromov as $\max \left\{\left|p_{i}\right|,\left|r_{i}\right|\right\} \rightarrow \infty$ for all $i=1, \ldots, d$, where $q_{i}=p_{i} / r_{i}$. Our main result shows that the Heegaard structure of the family of complete hyperbolic 3-manifolds of bounded volume strongly reflects this well-ordering property. We will show that generically, irreducible Heegaard splittings of uniformly bounded genus of the manifolds $M_{q}$, with $\max \left\{\left|p_{i}\right|,\left|r_{i}\right|\right\}$ sufficiently large for all $i=1, \ldots, d$, come from irreducible Heegaard splittings of $M$. This can be paraphrased as saying that irreducible Heegaard splittings of $M_{q}$ converge to such splittings of $M$ as $M_{q}$ converge to $M$. In other words the Heegaard structure is asymtotically "rigid" as are many other properties of these manifolds.

In this paragraph we define some terminology needed for our main theorem: Manifolds $M$ have a handle decomposition with one 0-handle. Let $g_{0}$ be the smallest number of 1-handles in such a decomposition of $M$. Notice that all manifolds obtained by Dehn surgery on $M$ have Heegaard splittings

\footnotetext{
${ }^{1}$ Partially supported by a grant from the German Israel Foundation for Research and Development (G.I.F.)

${ }^{2}$ Partially supported by a grant from the Australian Research Council.
} 
of genus $g_{0}$. If $g$ is an integer bigger than $g_{0}$ then all manifolds in $I M$ have irreducible Heegaard splittings of genus bounded above by $g$. A simple closed curve on a handlebody $H$ will be called a core curve of $H$ if it is isotopic to a simple closed curve in the spine of the handlebody which intersects some essential non separating disk in a unique point.

Our main theorem is:

\section{Theorem 0.1.}

a) There is a d-tuple of integers $\left(N_{1}, \ldots, N_{d}\right)$ defining a sub.collection $\mathbb{M}^{\prime}$ of $\mathbb{M}$ by $\mathbb{M}^{\prime}=\left\{M_{q} \in \mathbb{M} \mid \max \left(\left|p_{i}\right|,\left|r_{i}\right|\right)>N_{i}, i=1, \ldots, d\right\}$ and $a$ finite collection of surfaces $\Sigma^{1}, \ldots, \Sigma^{r}$ embedded in $M$ so that every irreducible Heegaard splitting surface of genus less than or equal to $g$ of manifolds $M_{q} \in \mathbb{M}^{\prime}$ is isotopic to one of the $\Sigma_{i}$.

b) A surface $\Sigma_{i}$ is either a Heegaard splitting for $M$ or there is at least one cusp and a unique simple closed curve $\beta$ on the boundary of a regular neighborhood of the cusp which is isotopic to a simple closed curve $\beta^{\prime}$ on $\Sigma_{i}$. In this case the curve $\beta^{\prime}$ is not isotopic to a core curve of any of the handlebodies determined by $\Sigma_{i}$.

Remark 0.2. Note that if $\Sigma_{i}$ is a Heegaard splitting for $M$ (i.e. bounding compression bodies) then there are many such curves $\beta$ on the boundary of a regular neighborhood of each cusp. If $\Sigma_{i}$ is not a Heegaard splitting and $\beta$ is chosen to be a $(0,1)$ element for some basis $\{\alpha, \beta\}$ of the cuspital homology of the cusp in question, only $(1, n)$ surgery on this cusp give manifolds in which $\Sigma_{i}$ corresponds to a Heegaard splitting (see [Sw]). Hence the corresponding Dehn surgery coordinate $q_{i}$ in $M_{q}$ must have been $(1, n)$.

Remark 0.3. In accordance with current terminology one can view the Heegaard splittings where the cusps cores of the compression bodies are "vertical", and the case where a cusp can be isotoped onto the surface but is not a core as "horizontal" Heegaard splittings. In this case we can paraphrase Theorem 0.1(b) as saying that bounded genus irreducible Heegaard splittings of the manifolds in $\mathbb{M}^{\prime}$ are either "vertical" or "horizontal". This is very similar to the situation in Heegaard splittings of Seifert fibered spaces (see [MS]).

Let $K$ be a knot in any 3-manifold $M$, in particular $S^{3}$. A tunnel system for $K$ is a collection of disjoint $\operatorname{arcs} t_{1}, \ldots, t_{s}$ properly embedded in $M-N(K)$ so that $M-N\left(K \cup\left\{\cup t_{i}\right\}\right)$ is a handlebody. Alternatively it is a collection of 
2-handles $t_{1}, \ldots, t_{s}$ so that $(M-N(K))-\left\{\cup \operatorname{int} t_{i}\right\}$ is a handlebody. If $K$ is a knot, it follows immediately that the handlebody is of genus $s+1$.

Definition 0.4. The tunnel number of a knot $K$, denoted by $t(K)$, is defined to be the minimal cardinality of all such collections $\left\{t_{1}, \ldots, t_{s}\right\}$.

As an application of Theorem 0.1 we have the following:

Definition 0.5. A subset of $\mathbb{Z} \oplus \mathbb{Z}$ will be called very simple if it is the union of a sub-sequence of elements of the form $\alpha+n \beta$ and a finite subset $A$ of $\mathbb{Z} \oplus \mathbb{Z}$, where $\{\alpha, \beta\}$ is a basis of $\mathbb{Z} \oplus \mathbb{Z}$ and $n \in \mathbb{Z}$. A subset of $\mathbb{Z} \oplus \mathbb{Z}$ will be called simple if it is the finite union of very simple sets.

Let $r=2 j+1, p_{1}, \ldots, p_{r}$ be odd integers so that $p_{i}=p_{r-i+1}$ and $K^{\prime}=K\left(p_{1}, \ldots, p_{r}\right)$ be a pretzel knot as in Fig. 1 with $r$ strands and $p_{i}$ crossings in the $i$-th strand. Assume also that g.c.d. $\left(p_{1}, \ldots, p_{r}\right)=p \neq 1$. Let $K^{\prime}(m / n)$ be the manifold obtained by $m / n$-Dehn surgery on $S^{3}-N\left(K^{\prime}\right)$. Theorem 0.6. Except for a simple subset of surgery coefficients $\{m / n\}$ the genus of $K^{\prime}(m / n)$ is $r$.

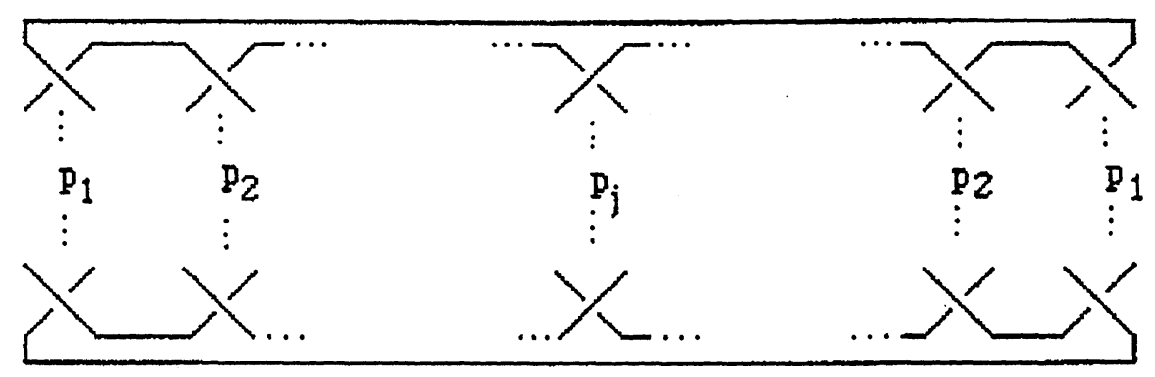

Figure 1.

If $K_{1}, K_{2} \subset S^{3}$ are two knots then $t\left(K_{1} \# K_{2}\right) \leq t\left(K_{1}\right)+t\left(K_{2}\right)+1$. This can be seen by considering a short unknotted tunnel on the decomposition 2 -sphere connecting the two points in which the composite knot intersects the 2-sphere.

The knots $K^{\prime}$ are all strongly invertible with respect to the involution $i:\left(S^{3}, K^{\prime}\right) \rightarrow\left(S^{3}, K^{\prime}\right)$ whose axis of symmetry is described in Fig. 2. It follows that the manifolds $K^{\prime}(m / n)$ are 2-fold branched covers of $S^{3}$ (see [Mo]). Let $K=K_{\left(K^{\prime}(m / n)\right)} \subset S^{3}$ be the branch set of this particular cover 
$M \rightarrow S^{3}$. If $m$ is odd then $K$ is a knot and if $m$ is even then $K$ is a two component link.

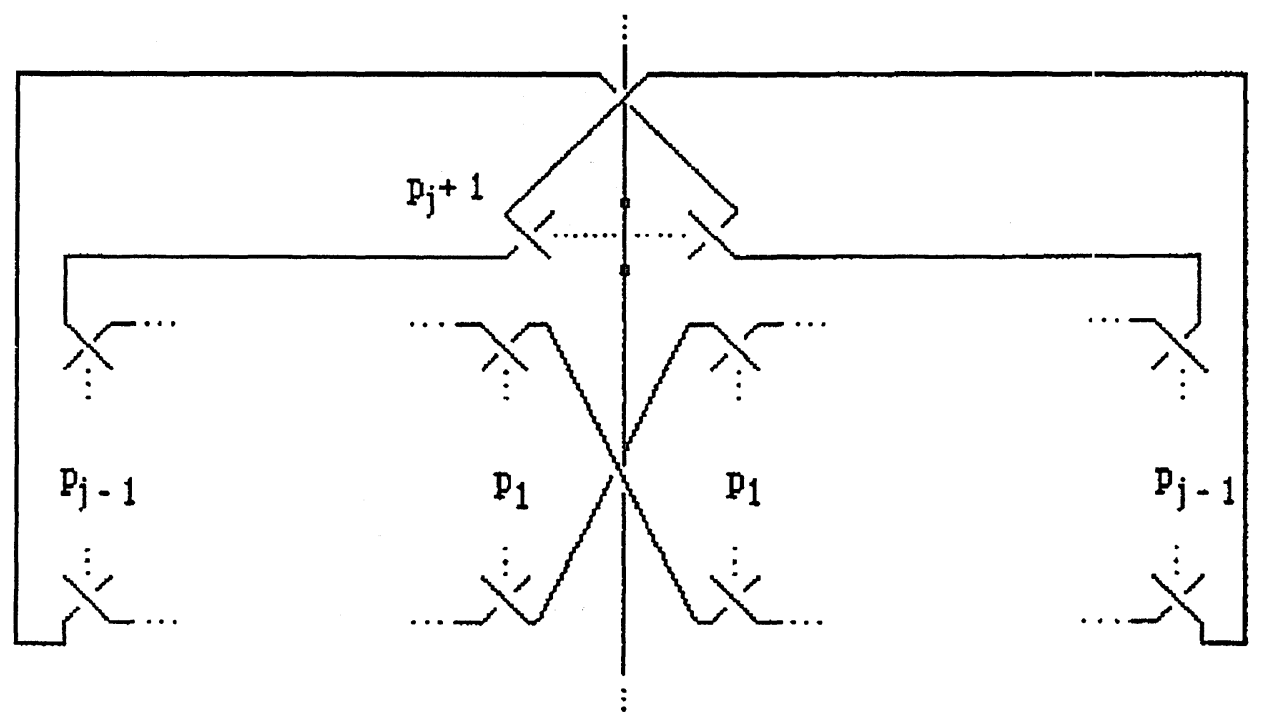

Figure 2.

Corollary 0.7. There exist infinitely many pairs of knots $K_{1}, K_{2}$ in $S^{3}$ which are branch sets corresponding, respectively, to pairs of pretzel knots $K^{\prime}\left(p_{1}, \ldots, p_{r}\right), K^{\prime}\left(p_{1}^{\prime}, \ldots, p_{s}^{\prime}\right)$, as defined above, with the property that

$$
t\left(K_{1} \# K_{2}\right)=t_{1}\left(K_{1}\right)+t\left(K_{2}\right)+1=(r+s) / 2 .
$$

Remark 0.8. Recently Morimoto Sakuma and Yokota (see [MSY]) using the examples of Corollary 0.7 have been able to show that for $K^{\prime}=$ $K^{\prime}(5,-5,5)$ we have $t(K)=1$ and $t(K \# K)=t(K)+t(K)+1=3$.

Acknowledgements. We would like to thank the departments of mathematics of the Technion at Haifa and the University of Melbourre for their generous hospitality. Also thanks to Joel Hass and Abby Thornpson and Marty Scharlemann for many conversations and the referee for his remarks regarding this work. 


\section{Negatively curved manifolds.}

Let $M$ be a hyperbolic 3-manifold of finite volume with $d$ cusps and $M^{\prime}$ be obtained from $M$ by removing fixed open horotorus neighborhoods of the cusps. Let $\left\{\gamma_{i, j}\right\}_{i=1}^{\infty}$ be sequences of geodesic curves on each component of $\partial M^{\prime}=\mathcal{T}_{j}$ with corresponding slopes $q_{i, j} \in \mathbb{Q} \cup\{\infty\}$. Let $M_{q_{i}}, q_{i}=$ $\left(q_{i, 1}, \ldots, q_{i, d}\right)$, be the manifold obtained from $M^{\prime}$ by doing Dehn surgery along the curves $\gamma_{i, 1}, \ldots, \gamma_{i, d}$. Denote the Euclidean length $\ell\left(\gamma_{i, j}\right)$ by $\ell_{i, j}$ and assume furthermore that $\ell_{i, j} \rightarrow \infty$. Let $|q|=\max \{|p|,|r|\}$, where $q=p / r$. Set $\left|q_{i}\right|=\min \left\{\left|q_{i, 1}\right|, \ldots,\left|q_{i, d}\right|\right\}$ and note that $\left|q_{i}\right| \rightarrow \infty \Leftrightarrow$ all $\ell_{i, j} \rightarrow \infty$.

The next Lemma is a generalization of Lemma 8 of $[\mathrm{BH}]$, see also Lemma 2.1 of [GT].

Lemma 1.1. For $\left|q_{i}\right|$ large enough there is a constant $C^{\prime}=C^{\prime}\left(\ell_{1}, \ldots, \ell_{d}\right)<$ 0 such that the manifold $M\left(q_{i}\right)$ has a metric $\rho_{q_{i}}$ of negative sectional curvature satisfying:

i) The metric $\rho_{q_{i}}$ restricted to $M^{\prime}$ is the original hyperbolic metric.

ii) The sectional curvatures of $\rho_{q_{i}}$ are bounded between $C^{\prime-1}$ and $C^{\prime}$.

iii) Furthermore $C^{\prime} \rightarrow-1$ as $\ell_{i, j} \rightarrow \infty$.

Corollary 1.2. There is a constant $C<0$ such that for sufficiently large $\left|q_{i}\right|$ the sectional curvatures of all $\rho_{q_{i}}$ are bounded between $C^{\prime-1}$ and $C^{\prime}$.

Proof. For each $q_{i}$ and each cusp we will define a negatively curved metric on a solid torus $V$ which is hyperbolic near $\partial V$ and then glue $\partial V$ to $\partial M^{\prime}$ so that the boundary of a meridional disk $D$ of $V$ is glued to $\gamma_{i, j}$. This can be done if the values and derivatives of the metrics on $M^{\prime}$ and $V$ agree on $\mathcal{T}$ and $\partial V$. The original hyperbolic metric on $M^{\prime}$ has a symmetric form which can be written in cylindrical coordinates in the neighborhood $N$ of the cusp as:

$$
d s^{2}=d r^{2}+f_{0}(r) d \gamma^{2}+g_{0}(r) d \alpha^{2}
$$

where $r \leq 0$ is the radial distance measured towards the cusp, $0 \leq \gamma \leq 1$ measures the distance in the direction of the geodesic curve $\gamma_{i, j}$ and $0 \leq$ $\alpha \leq 1$ measures the distance in a direction perpendicular to $\gamma_{i, j}$. In this form $f_{0}(r)=\ell_{i, j} e^{r}$ and $g_{0}(r)=\ell e^{r}$ where $\ell$ is the length of a fixed arc in a direction perpendicular to $\gamma_{i, j}$ meeting $\gamma_{i, j}$ at its ends only, and $\ell_{i, j}$ is the length of $\gamma_{i, j}$. For the convenience of notation, since only one cusp at a time needs to be considered, we drop the suffix $j$ so $\ell_{i}=\ell_{i, j}$. 
We seek a metric $d s$ on $V$ of the form $d s^{2}=d r^{2}+f(r) d \gamma^{2}+g(r) d \alpha^{2}$ so that $f, f_{0}$ and $g, g_{0}$ agree smoothly on $\partial V$. Note that now we need to measure $r$ the radial distance of a meridional disk of $V$ from the core outwards so $r=$ 0 corresponds to the core of $V$. With this choice of coordinates the sectional curvatures are convex combinations of $K(r, \gamma)=-f^{\prime \prime} / f, K(r, \alpha)=-g^{\prime \prime} / g$ and $K(\gamma, \alpha)=-f^{\prime} g^{\prime} / f g$ (see $\left.[\mathrm{BH}]\right)$. If $f(0)=0$ then the cone angle around the core of $V$ is

$$
\lim _{r \rightarrow 0} \frac{1}{r} \int_{0}^{1} f(r) d \gamma=f^{\prime}(0)
$$

Hence we want functions $f(r)$ and $g(r)$ with the following properties:

i) $f(0)=0, f\left(\log \ell_{i}\right)=\ell_{i}$,

ii) $f^{\prime}(0)=2 \pi$ to ensure that the metric is non-singular around the core of $V, f^{\prime}\left(\log \ell_{i}\right)=\ell_{i}$,

iii) $f^{\prime \prime} / f \rightarrow 1$, when $i \rightarrow \infty$,

iv) $g\left(\log \ell_{i}\right)=\ell$,

v) $g^{\prime}\left(\log \ell_{i}\right)=\ell$,

vi) $g^{\prime \prime} / g \rightarrow 1$, when $i \rightarrow \infty$,

vii) $f g^{\prime} / f g \rightarrow 1$, when $i \rightarrow \infty$.

Note that the metric is constructed so that for all $i$ the radius of the meridional disk of $V$ is $\log \left(\ell_{i}\right)$.

Assume that we have found a function $f$ with the desired properties and consider the equation

$$
g^{\prime} / g=f / f^{\prime}
$$

hence $\frac{d \log g}{d r}=f / f^{\prime}$ so $\log g=\int \frac{f}{f^{\prime}}+C$ and $g=A e^{\int \frac{f}{f^{\prime}}}$. Set $A=\frac{\ell}{\ell_{i}}$ so that $g\left(\log \ell_{i}\right)=\ell$. Note also that $g^{\prime}=g f / f^{\prime}$ hence $g^{\prime}\left(\log \ell_{i}\right)=g\left(\log \ell_{i}\right)$. Furthermore by differentiating equation (1) we get:

$$
g^{\prime \prime} / g-\left(g^{\prime} / g\right)^{2}=1-f f^{\prime \prime} /\left(f^{\prime}\right)^{2}=\left(f / f^{\prime}\right)^{2} f^{\prime \prime} / f
$$

so

$$
g^{\prime \prime} / g=1+\left(f / f^{\prime}\right)^{2}\left(1-f^{\prime \prime} / f\right)
$$


Hence if $\left(f / f^{\prime \prime}\right)^{2}$ is bounded and if $f^{\prime \prime} / f \approx 1$ then $g^{\prime \prime} / g \approx 1$. Set $f(r)=$ $e^{r}+h(r)$ where $h(r)=\frac{2 \pi-1}{\log \ell_{i}}\left(r-\frac{\log \ell_{i}}{2}\right)^{2}+\frac{(2 \pi-1) \log \ell_{i}}{4}-1$ for $0 \leq r \leq \log \ell_{i}-1$. For $\log \ell_{i}-1 \leq r \leq \log \ell_{i} h(r)$ is equal to a strictly decreasing smooth function $a(r)$ such that:

i) $a^{(n)} \log \left(\ell_{i}-1\right)=h^{(n)} \log \left(\ell_{i}-1\right), 0 \leq n \leq 2$,

ii) $a^{(n)} \log \left(\ell_{i}\right)=0,0 \leq n \leq 2$,

iii) $\sup \left|a(r) / a^{\prime}(r)\right|<C^{\prime}$ for $\log \ell_{i}-1 \leq r \leq \ell_{i}$ and some constant $C^{\prime}$,

iv) $\sup \left|a^{\prime \prime}(r) / a(r)\right|<C^{\prime \prime}$ for $\log \ell_{i}-1 \leq r \leq \ell_{i}$ and some constant $C^{\prime \prime}$.

It is a standard argument that such functions exist (see Fig. 3). Since $h\left(\log \ell_{i}-1\right)=2 \pi-2-(2 \pi-1) / \log \ell_{i} \rightarrow 2 \pi-2$ and $h^{\prime}\left(\log \ell_{i}-1\right)=$ $-(2 \pi-1)\left(1-2 / \log \ell_{i}\right) \rightarrow-(2 \pi-1)$ as $\ell_{i} \rightarrow \infty$ i.e., $i \rightarrow \infty$ we can find some integer $N_{0}$ such that for all $i$ larger than $N_{0}$ we can choose fixed constants $C^{\prime}$ and $C^{\prime \prime}$.

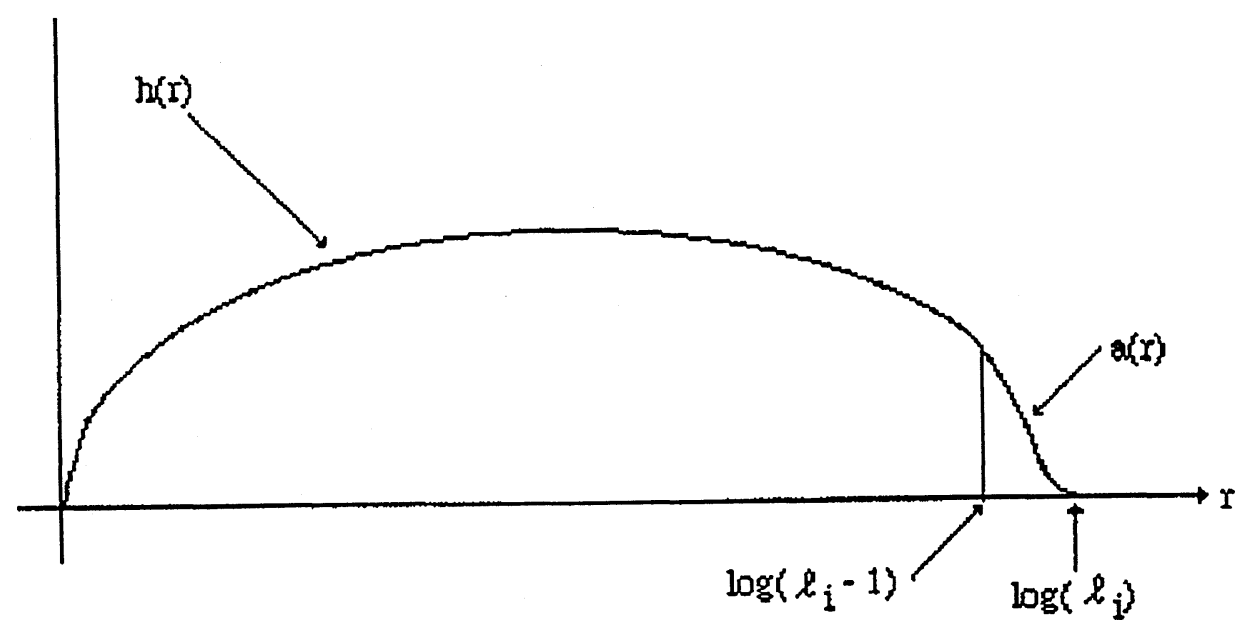

Figure 3.

It is straight forward to check that the function $f(r)$ has the desired properties (i)-(vii) and $\left(f / f^{\prime}\right)^{2}$ is bounded. So we can find a constant $C\left(\ell_{i}\right)$ satisfying the lemma for some $N^{\prime}$ large enough.

Lemma 1.3. For each cusp the area of meridional disks $D$ in the surgery tori $V$ grows arbitrarily large as the length of boundary $\partial D=\ell$ goes to infinity. 
Proof. Consider now the minimal disk $D$ of the surgery solid torus $V$. Again by the Gauss-Bonnet Theorem for bounded surfaces we have:

$$
\int_{\partial D} k_{g} d s+\int_{D} K_{i} d A=2 \pi \mathcal{X}(D)=2 \pi
$$

where $k_{g}$ is the geodesic curvature of $\partial D$ and $s$ is arc length. Now $\partial D$ is a geodesic in the metric of the boundary horotorus $\mathcal{T}$ which is flat and $D$ is perpendicular to $\mathcal{T}$. It follows that $k_{g}=1$. Hence

$$
\int_{\partial D} k_{g} d s=\ell_{i}
$$

so

$$
2 \pi-\int_{D} K_{i} d A=\ell_{i}
$$

but

$$
2 \pi-\int_{D} K_{i} d A<2 \pi-\int_{D} C^{-1} d A=2 \pi-C^{-1} \operatorname{Area}(D)
$$

i.e., $\operatorname{Area}(D) \rightarrow \infty$ as $\ell_{i} \rightarrow \infty$. Note that by definition, any other choice of meridional disk $D^{\prime}$ coming, say, from a general minimal surface will have larger area than $D$.

Corollary 1.4. A closed orientable embedded minimal surface $\Sigma$ of genus $g$ in $M_{q_{i}}$ cannot intersect any surgery solid torus $V$ in any meridional disk if $\left|q_{i}\right|$ is sufficiently large.

Proof. If the surface $\Sigma$ intersects some surgery solid torus $V$ in some meridional disk $D^{\prime}$ and $\left|q_{i}\right|$ is sufficiently large then $\ell_{i}$ is sufficiently large and it follows from the proof of Lemma 1.3 that $\operatorname{Area}(D)$ is bigger than any constant, where $D$ is the meridional disk. However $\operatorname{Area}(\Sigma)>\operatorname{Area}\left(D^{\prime}\right) \geq \operatorname{Area}(D)$. But by the Gauss-Bonnet theorem, since the curvatures of all surfaces are uniformly bounded, Area $(\Sigma)$ is uniformly bounded by $2 \pi(2-2 g) / C$ so we have a contradiction.

Definition 1.5. Let $\Sigma$ be an embedded minimal surface in $M$. Given two horotori in a cusp which are a distance $t$ apart, suppose that $\Sigma$ intersects 
the region of $M$ in between the two horotori in a spanning annulus $T$. Then $T$ will be called a tube of length $t$ with core $\alpha$ if a generating loop $\alpha$ is contractible in $M$.

Lemma 1.6. There is a number $t$ so that length of tubes in $M$ is uniformly bounded above by $t$.

Proof. Consider the universal cover $\widetilde{R}$ of the region $R$ between the two horotori. Lift a tube $T$ to a surface $\widetilde{T}$ in $\widetilde{R}$ and consider a ball $B$ of radius $t / 2$ about a lift of a point in $T$ that is midway between the horospheres of $\partial \widetilde{R}$. By the Monotonicity Formula (see [Si]) there are constants $\beta, \gamma \in \mathbb{R}$ such that $\operatorname{Area}(\widetilde{T} \cap B) \geq \beta e^{\gamma t}$, which is the area of a totally geodesic hyperbolic disk of radius $t / 2$. However by the Gauss-Bonnet theorem and Corollary 1.2 , the area of the surface $\Sigma$ is uniformly bounded by $2 \pi(2-2 g) / C$, hence the result follows.

\section{On some bounded surfaces in solid tori.}

Let $\Sigma$ be a Heegaard surface of genus $g$ of a closed 3-manifold $M$ separating $M$ into two handlebodies $H$ and $H^{\prime}$. We will say that $\Sigma$ is strongly irreducible if every compressing disk for $\Sigma$ in $H$ intersects every compressing disk for $\Sigma$ in $H^{\prime}$. Let $V$ be a non trivial solid torus in $M$ (i.e. $\pi_{1}(V)$ injects into $\pi_{1}(M)$ ). Assume that $\Sigma$ intersects $\partial V$ only in essential curves on $\partial V$ which do not bound meridian disks of $V$. Denote the surface $\Sigma \cap V$ by $F$ and for convenience assume first that $\Sigma \cap M^{\prime}$ contains no annuli which are parallel to $\partial M^{\prime}$, where $M^{\prime}=M-\operatorname{int} V$.

Lemma 2.1. The surface $F$ has at most one component $F^{\prime}$ which is not an annulus.

Proof. We may assume that the surface $F=\Sigma \cap V$ is isotopic in $V$ to a collection of annuli $\mathcal{A}$ connected perhaps by tubes. If $F$ is incompressible in $V$ we are done as the only incompressible surfaces in a solid torus are either disks or annuli and by the assumption on $F$ we cannot have essential disks. If not, compress $F$ in $V$ as much as possible and the resulting surface is a collection of annuli. We can reconstruct $F$ from these annuli by deleting disks and replacing them by tubes (annuli). It follows that the number of boundary components of $F$ is even, say $2 r$.

Since $\Sigma$ separates $M$ the surface $F$ separates $V$ into components in either $H$ or $H^{\prime}$ so we have a well defined notion of two sides of $F$. Furthermore 
note that all the innnermost compression disks of the tubes (i.e. compression disks for $F$ which do not intersect $F$ in their interior) must be on one side of $F$ as otherwise we contradict the fact that the Heegaard splitting is strongly irreducible.

Note next that the surface $F$ splits $V$ into handlebodies as $\partial V-F$ is a collection of essential annuli $A_{1}, \ldots, A_{r}$ and the handlebodies $H$ and $H^{\prime}$ cut along essential annuli split into handlebodies. Each of the annuli $A_{i}$ is contained in one of the two handlebodies $H$ or $H^{\prime}$ and boundary compresses there. Let $H$ denote the handlebody which contains the innermost compression disks for the tubes. Choose an outermost annulus $A_{i}$ in the other handlebody $H^{\prime}$ and a boundary compression disk $D$ for it. The boundary of the disk $D$ is the union of two $\operatorname{arcs} \lambda \cup \alpha$ where $\lambda$ denotes the $\operatorname{arc} D \cap \Sigma$ and $\alpha$ is on $A_{i}$. Note that $D$ can be a priori on either side of $\partial V$.

We claim that the disk $D$ must be contained in the solid torus $V$. As otherwise after performing the boundary compression for the annulus $A_{i}$ along $D$ we will obtain a disk $D^{*}$ in $H^{\prime}$ : Two boundary curves of $F^{\prime}$ become connected along a trivial short band in $H^{\prime} \cap \partial V$, giving an essential compressing disk for $F^{\prime}$ and $H^{\prime}$ i.e. a compressing meridian disk for a tube. Since we assumed that $\Sigma \cap M^{\prime}$ contains no annuli which are parallel to $\partial M^{\prime}$ the disk $D^{*}$ must be essential in $H^{\prime}$. We have disjoint innermost tube disks in $H$ at the same time, violating the strong irreducibility of the splitting. Hence $D$ is contained in $V$ and the $\operatorname{arc} \lambda$ is equal to $D \cap F$. Furthermore the $\operatorname{arc} \lambda$ must intersect all innermost tubes of $F$ as otherwise after doing the boundary compression along $D$ for $A_{i}$, this time in $V$, we will have disjoint essential (meridian) disks for both $H$ and $H^{\prime}$ on either side of $F$ in contradiction to the assumption that $\Sigma$ is strongly irreducible. Since $\lambda$ does not meet $\partial V$ other than at its end points and hence is contained in one component of $F$, all innermost tubes are on the same component $F^{\prime}$ of $F$ as $\lambda$.

Assume now that there is some other component of $F$ which is not an annulus. Consider an innermost tube for the surface $F-F^{\prime}$ and let $F^{\prime \prime}$ denote the component of $F$ it belongs to. Let $D^{\prime \prime}$ be a compressing disk for this tube, so chosen that the number of components of intersection of $D \cap D^{\prime \prime}$ is minimal. We cannot have $D \cap D^{\prime \prime}=\varnothing$ as $D^{\prime \prime}$ is not innermost so there is an innermost disk contained in $D^{\prime \prime}$ and $\lambda \subset \partial D$ intersects all innermost disks so $\lambda \cap D^{\prime \prime} \neq \varnothing$. If we have loops and trivial arcs of intersection (i.e. $\operatorname{arcs}$ in $D^{\prime \prime}$ with both end points on the same components of $F^{\prime \prime}$ bounding a disk in $D^{\prime \prime}$ ) we can change $D^{\prime \prime}$ by cut and paste techniques to reduce the number of intersection components. Therefore there must be an essential arc of intersection on $D$. The situation is indicated schematically in Fig. 4 below. Choose an outermost such arc on $D$ and denote it by $\gamma$. The 
arc $\gamma$ together with a sub-arc of $\lambda$ bounds a sub-disk $D_{0}$ of $D$. Now modify $D^{\prime \prime}$ by removing a regular neighborhood $\gamma \times I$ of $\gamma$ from $D^{\prime \prime}$ and gluing instead the boundary of a regular neighborhood of $D_{0}=\partial N\left(D_{0}\right)-\operatorname{int}(\gamma \times I)$.

This also reduces the number of components of intersection which is a contradiction to the choice of $D^{\prime \prime}$. Hence we cannot have non-annular components other than $F^{\prime}$.
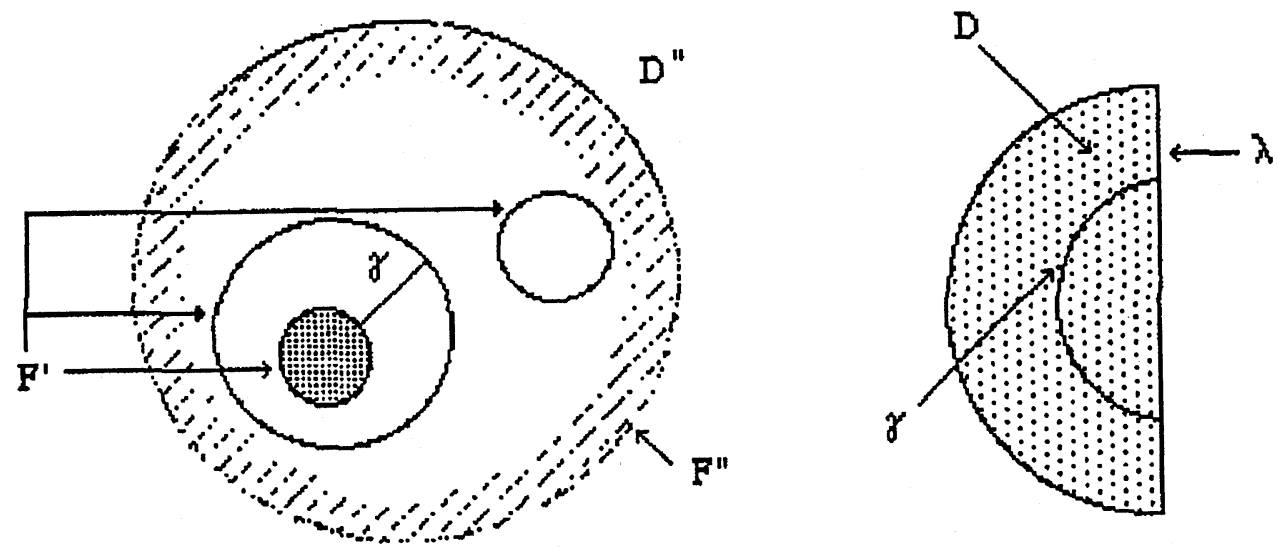

Figure 4 .

In the next lemma we deal with the possibility that the tubes in $F^{\prime}$ might be nested.

Lemma 2.2. The non-annular component $F^{\prime}$ of $F$ is isotopic to a collection of cyclically ordered annuli $\mathcal{A}$ in $V$ (i.e. no annuli are nested) connected by tubes. Furthermore there is a complete system of compressing disks for the tubes of $F^{\prime}$ so that the interior of the disks is disjoint from $F^{\prime}$.

Proof. Choose a system of compressing disks $\left\{D_{1}, \ldots, D_{s}\right\}$ for $F^{\prime}$ so that the intersection of the interior of the $D_{i}$ 's and the boundary compressing disk $D$ of $A_{i}$, as in the previous lemma, is minimal. If the intersection is empty we are done with the second part of the claim, so assume that it is not empty. Recall that $\partial D$ intersects every innermost tube in $F^{\prime}$ and hence $\partial D$ intersects every one of the innermost $D_{i}$ 's. By an easy cut and 
paste argument we can assume that the intersection does not contain trivial arcs and loops i.e., arcs and loops which bound disks on both surfaces.

Choose an outermost arc of intersection $\gamma$ on $D$. We have two possible configurations depending on whether the arc $\gamma$ connects a disk to itself (Fig. 5 case (a)) or two disks to each other (Fig. 5 case (b)). In both cases we replace a small regular neighborhood of $\gamma$ in the disk $D_{j}$ denoted $N(\gamma)$ by the boundary of a regular neighborhood of the sub-disk of $D$ bounded by $\gamma$ and a sub-arc of $\lambda$, less $N(\gamma)$. We now have a new disk $D_{j}^{\prime}$ with fewer components of intersection between its interior and the interior of $D$. This contradicts the choice of the disks $\left\{D_{1}, \ldots, D_{s}\right\}$, (see Fig. 5 below). Hence no compressing disk can intersect an innermost tube, which is the second part of the claim.

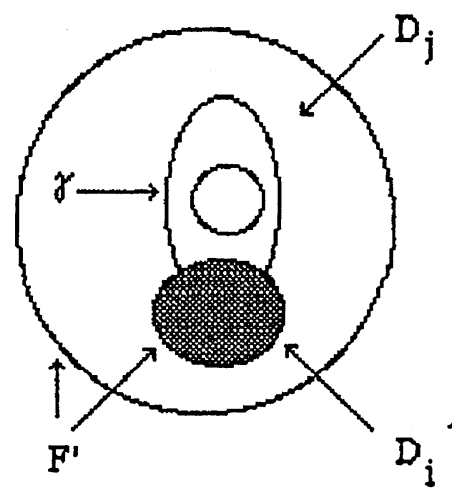

(a)

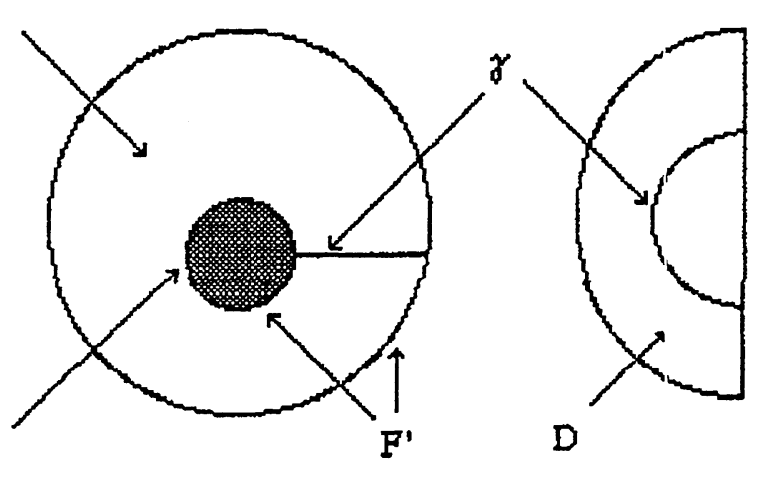

(b)

Figure 5 .

If the compressing disks $\left\{D_{1}, \ldots, D_{s}\right\}$ are disjoint from $F^{\prime}$ then we cannot have nested annuli. In order for annuli to be nested we must have at least three annuli and there must be some annulus in $V$ with annuli on both sides. As $F^{\prime}$ is connected we must have tubes connecting the annuli and since the compressing disks $\left\{D_{1}, \ldots, D_{s}\right\}$ are disjoint from $F^{\prime}$ we have essential disks on both sides of $F^{\prime}$ thus contradicting the strong irreducibility of $\Sigma$. Thus the annuli are cyclically ordered around $V$, as indicated in Fig. 6 below where we have drawn a cross section of the torus $V$ with the annuli 
and tubes with their meridian compressing disks indicated.
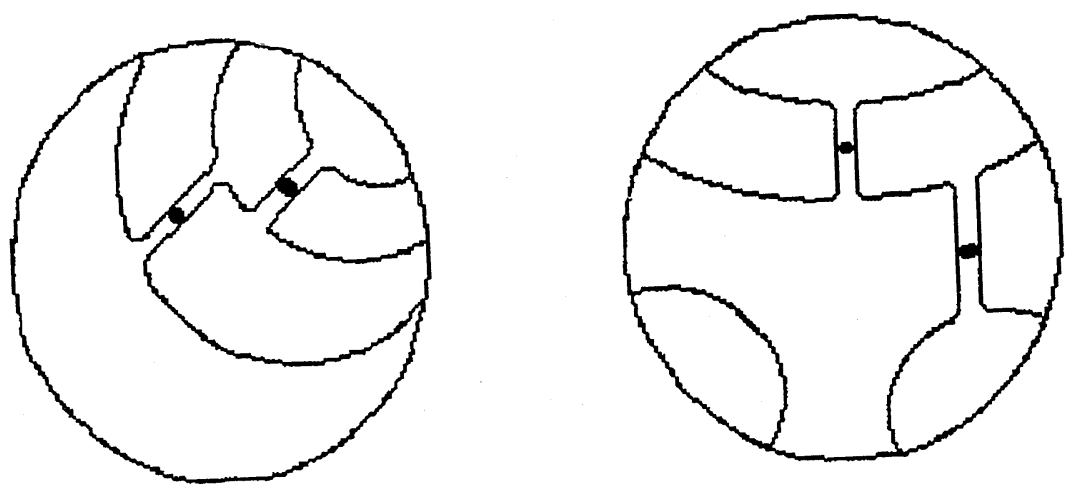

Figure 6.

Proposition 2.3. The surface $F^{\prime}$ is isotopic to one annulus with one standard tube attached or to two annuli connected by one standard tube.

Here, the tube is standard if there is a boundary compression disk $D \subset V$ for $F^{\prime}$ so that $\partial D$ meets a meridian compressing disk for the tube in exactly one point. (So the tube is "boundary parallel".)

Proof. If the surface $F^{\prime}$ is isotopic to either one annulus with one standard tube attached or to two annuli connected by one standard tube we are done so we assume that $F^{\prime}$ has either three annuli or less than three annuli but at least two tubes. In any case the genus of both handlebodies $H \cap V$ and $H^{\prime} \cap V$ is greater or bigger than three. The proof is divided into three cases depending on the slope of the curves of $\partial F^{\prime}$.

Case 1. The curves of $\partial F^{\prime}$ are isotopic to the core of $V$.

We claim that the surface $F^{\prime}$ also looks like a collection of tubes attached to cyclically ordered annuli $\mathcal{A}$ in $H^{\prime}$. To be precise, in Lemma 2.2 we showed that if an innermost compression of a tube of $F^{\prime}$ in $H$ is in the interior of $V$, then $F^{\prime}$ looks like cyclically ordered annuli plus tubes relative to $H$.

The annuli $A_{1}, \ldots, A_{r}$ which are in $H^{\prime}$ also boundary compress. After doing a boundary compression, two boundary curves of $F^{\prime}$ become connected along a trivial short band in $H^{\prime} \cap \partial V$, giving an essential compressing disk 
for $F^{\prime}$ in $H^{\prime}$ i.e. a compressing meridian disk for a tube. If we push this compressing disk slightly into $V$ (off $\partial V$ ) we obtain a compressing disk for $F^{\prime}$ in $H^{\prime}$ in the interior of $V$. So this tube also existed, in the part of $H^{\prime}$ contained in $V$, before the boundary compression. Hence we could have found a collection of compressing disks for innermost tubes, as in Lemma 2.1, in $H^{\prime}$. Therefore, using Lemma 2.2, up to isotopy $F^{\prime}$ can also be expressed as a surface $F^{\prime \prime}$ which is a collection of tubes attached to cyclically ordered annuli in $H^{\prime}$.

Consider an isotopy $F_{t}^{\prime}$ of $F^{\prime}$, for $0 \leq t \leq 1$, taking $F^{\prime}$ to $F^{\prime \prime}$ with $F_{0}^{\prime}$ consisting of annuli of $H \cap \partial V$ with thin tubes attached and $F_{1}^{\prime}=F^{\prime \prime}$ equal to the annuli of $H^{\prime} \cap \partial V$ with thin tubes attached. Let $\mathcal{D}$ be a meridian disk for the solid torus $V$. The isotopy $F_{t}^{\prime}$ can be chosen to be in general position relative to $\mathcal{D}$, so that there is a finite number of critical levels $0<t_{1}<\cdots<t_{s}<1$, where $F_{1}^{\prime} \cap \mathcal{D}$ has a single non-degenerate critical point, which is either a saddle, a maximum or a minimum. Some values of $t: 0, t_{i}-\varepsilon, t_{i}+\varepsilon, 1$ of $F_{t}^{\prime} \cap \mathcal{D}$ are shown schematically in Fig. 7 .

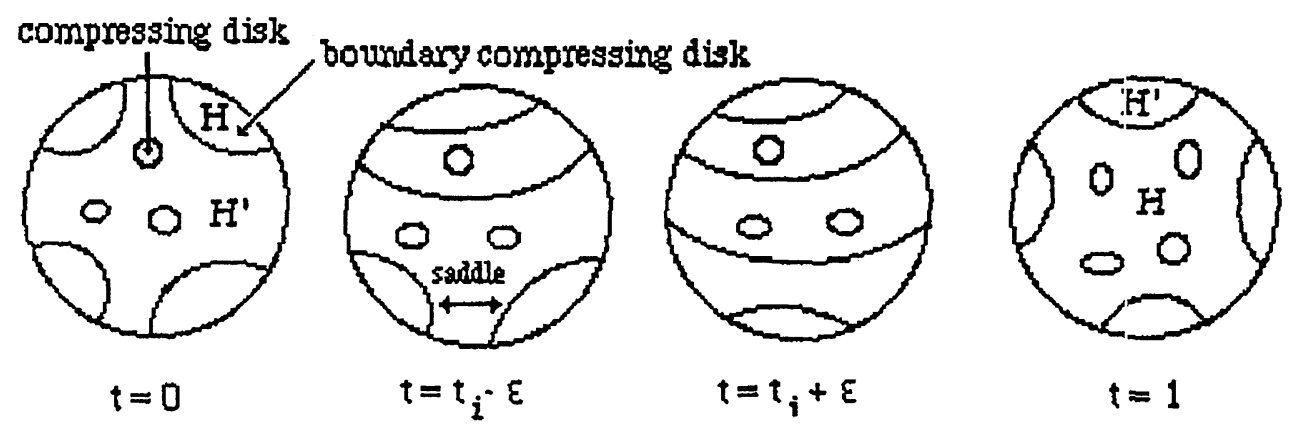

Figure 7.

Note that for $t=0$, all the innermost disks in $\mathcal{D}$ (as in Fig. 7) representing compressing meridian disks for tubes and outermost 2-gons in $\mathcal{D}$ (as in Fig. 7) representing outermost boundary compressing disks for the annuli must be in $H$, whereas for $t=1$ must all be in $H^{\prime}$. As the isotopy proceeds we can never have such disjoint meridian disk or an outermost boundary compressing disk simultaneously both in $H$ and in $H^{\prime}$, since this would contradict strong irreducibility. (Note that we ignore innermost disks whose boundary circles are inessential on $F^{\prime}$. Also an outermost boundary compressing disk gives a meridian disk for $H$ or $H^{\prime}$ by boundary compressing $F^{\prime}$ as above.) Consequently at some critical point $t_{i}$, all the innermost compressing meridian disks and outermost boundary compression disks must 
"flip over" from $H$ to $H^{\prime}$. The level $t_{i}$ is a single saddle, maximum or a minimum.

This is possible if $F^{\prime}$ has two or four boundary curves giving one or two outermost boundary compression disks. For if there are three or more outermost boundary compression disks, at least one cannot be changed at the critical point $t_{i}$ thus contradicting strong irreducibility. By Lemma 2.2 $F^{\prime}$ is a collection of cyclically ordered annuli connected by tubes so each annulus contributes a outermost boundary compression hence there can be at most two such annuli. If there are two such annuli they must be connected by a tube as $F^{\prime}$ is connected.

It remains to prove that the tube is standard:

If $F^{\prime}$ has four boundary curves at the critical point $t_{i}$ then all simple closed curves of $F^{\prime} \cap \mathcal{D}$ must be inessential on $F^{\prime}$. This is because the critical point must involve the outermost boundary compression disks, so the loops remain unchanged (hence cannot be essential). We can pull all the loops off $\mathcal{D}$, and then $F^{\prime} \cap \mathcal{D}$ has exactly two arcs (see Fig. 9(b)).

If the case that $F^{\prime}$ has two boundary curves, $F^{\prime} \cap \mathcal{D}$ has exactly one arc. At the critical level, essential innermost disks of $F^{\prime} \cap \mathcal{D}$ must "flip over". There can be at most one such disk. If there are more then two innermost disks then at the critical level a saddle connecting at most two innermost disks in $H$, say, will still give an innermost disk in $H$. In this situation, if this disk is essential in $H$ we get a contradiction to strong irreducibility, if the result is an inessential disk we can conclude that there is a level where all loops are inessential on $F^{\prime}$ and can be pushed off $\mathcal{D}$.

If there is one essential innermost disk, we would get the possibility as in Fig. 8. But then the outermost boundary compression disk $D$ is disjoint from the essential innermost disks before and after the critical point and this contradicts strong irreducibility.

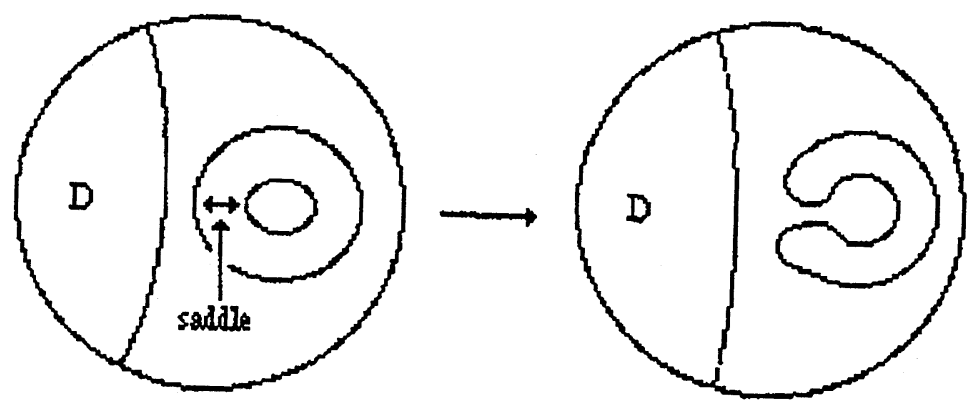

Figure 8. 
We conclude that $F^{\prime} \cap \mathcal{D}$ is either one or two arcs, for a suitable choice of $\mathcal{D}$. Now cut $V$ open along $\mathcal{D}$ to get a 3-ball $B$. Let $F^{\prime} \cap B$ denoted by $F^{*}$ (See Fig. 9. The indicated handle could be a priori knotted.)

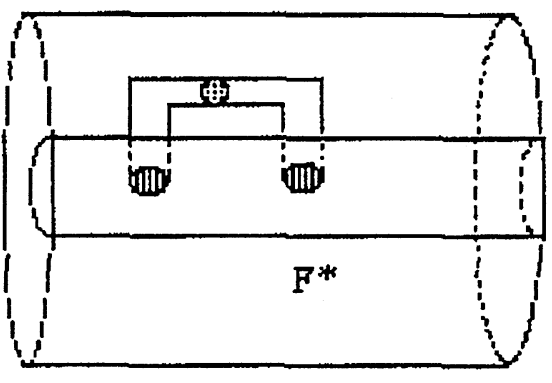

B

(a)

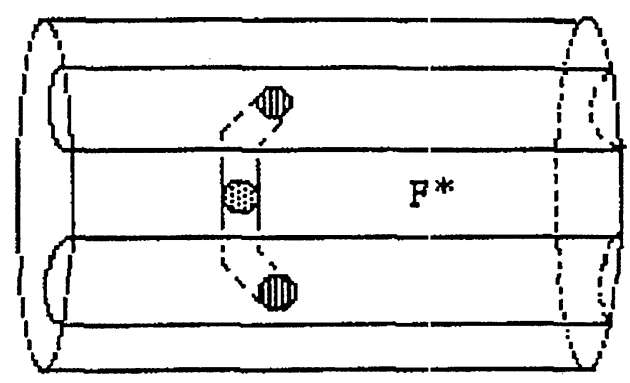

B

Figure 9.

In Fig. 9(a), $\partial F^{*}$ is a single curve. The surface $F^{*}$ separates the 3 -ball $B$ into two handlebodies as $\mathcal{D}$ intersects the handlebodies $H$ and $H^{\prime}$ in a collection of disks (see Fig. 9). By Waldhausen's theorem on Heegaard splittings of $S^{3}$, [Wa1], since $F^{*}$ is a strongly irreducible Heegaaird surface (as $F^{\prime}$ is) we conclude that $F^{*}$ must be a disk. Hence the original surface $F^{\prime}$ is an annulus.

In Fig. 9(b), we argue exactly as in Lemma 2.1, by strong irreducibility of $F^{*}$, that the annulus component of $\partial B-\partial F^{*}$ must be boundary compressible and the boundary compressing disk must be inside of $B$ rather than outside. After doing this boundary compression on $F^{*}$ the surface $F^{*}$ is replaced by a surface $\widetilde{F}$ which has the property that $\partial \widetilde{F}$ has a single component and $\widetilde{F}$ defines a Heegaard splitting of $B$ (as the boundary compression cuts the handlebodies only along disks). Again we can apply Waldhausen's theorem to show that $\widetilde{F}$ must be a disk. Consequently, (reconstructing $F^{*}$ by gluing along arcs) $F^{*}$ is an annulus i.e. a pair of disks joined by a standard tube and $F^{\prime}$ is two annuli connected by a boundary parallel tube. This completes case 1 .

Case 2. The boundary curves of $F^{\prime}$ are isotopic to a $p, q$-curve on $\partial V,|p|>1$, and one of the annuli, say $\mathcal{A}$, resulting from compressing the tubes of $F^{\prime}$, on either the $H$ or the $H^{\prime}$ side, is such that the solid torus component $V_{1}$ of $V-\mathcal{A}$ containing the compression disk for $\mathcal{A}$ has $\operatorname{int} V_{1} \cap F^{\prime} \neq \varnothing$.

Cut $V$ open along $\mathcal{A}$. Consider the component $V_{1}$ of $V-\mathcal{A}$ containing $F^{\prime}$. Then $F^{\prime}$ in $V_{1}$ satisfies the conditions of Case 1 . This is because $\mathcal{A}$ is 
parallel to the closure of $\partial V_{1}-\mathcal{A}$ in $V_{1}$ by assumption, so the curves of $\partial \mathcal{A}$ and $\partial F^{\prime}$ are isotopic to the core of $V_{1}$. Hence by Case $1 F^{\prime}$ is isotopic to one annulus or to two annuli connected by one standard tube. The surface $F^{\prime}$ is not affected when we glue $V_{1}$ back along $\mathcal{A}$ to $V-V_{1}$. So we obtain a surface in $V$ of the desired type. Only in this case $\partial F^{\prime}$ are $p, q$-curves. This completes Case 2 .

Case 3. The boundary curves of $F^{\prime}$ are isotopic to a $p, q$-curve on $\partial V,|p|>1$, and every one of the annuli $\mathcal{A}$ resulting from compressing the tubes of $F^{\prime}$, on either the $H$ or the $H^{\prime}$ side, is such that the solid torus component $V_{1}$ of $V-\mathcal{A}$ containing the compression disk for $\mathcal{A}$ has int $V_{1} \cap F^{\prime}=\varnothing$. We will call such annuli $\mathcal{A}$ boundary parallel away from $F^{\prime}$.

We use an argument similar to the argument of $[\mathrm{BO}]$ in the classification of Heegaard splittings of lens spaces.

Fiber the torus $V$ so that the curves of $\partial F^{\prime}$ are regular fibers. Let $\sigma$ denote the singular fiber in $V$. A singular annulus $A_{0}$ is obtained from an annulus $A$ by a $p$-fold identification of points on one of the boundary components of the annulus. Choose an embedding of a singular annulus $A_{0}$ into $V$. The embedding in $V$ is selected so that $A_{0} \cap \partial V$, the non-singular component of $\partial A_{0}$, is a regular fiber disjoint and parallel to the curves of $\partial F^{\prime}$. The other, singular component of $\partial A$ is mapped to the curve $\sigma$ which is a core curve for $V$.

Since all the annuli $\mathcal{A}$ are boundary parallel away from $F^{\prime}$ the Heegaard splitting of $V$ determined by $F^{\prime}$ determines a height function $h: V \rightarrow[0,1]$ with the following properties:

1. $h^{-1}(1 / 2)=F^{\prime}$.

2. $h^{-1}(0)=Q, h^{-1}(1)=Q^{\prime}$ where $Q$ is a spine of the annuli of $H \cap \partial V$ and $Q^{\prime}$ is a spine of the annuli of $H^{\prime} \cap \partial V$.

3. $h$ has $g$ critical points of index one and $g$ critical points of index two, where $F^{\prime}$ has $g$ tubes.

4. $h$ has no critical points of index zero or three.

The height function $h: V \rightarrow[0,1]$ induces a foliation by level curves on $A_{0}$ and hence by pull back on $A$. By general position we can isotope $A_{0}$ so that the curve $\sigma$ lies in the collar between the critical points of index one and two, as in [BO].

We can isotope $\sigma$ to lie in a level surface of $h$ hence in $F^{\prime}$ (here the annulus $A$ fills the role of the disk $\Delta$ in [Bon] and [BO]) by a straightforward 
application of the argument in Lemma 3 of [BO]: An easy way to think of this argument is to add a solid torus $V^{\prime}$ to the boundary of $V$ to form a Lens space. The solid torus $V^{\prime}$ has a meridian disk $D$ with $\partial D$ parallel to the boundary of $A_{0}$ and to $F^{\prime} \cap V$. Hence $A_{0} \cup D$ is a spine of the resulting Lens space. Note that the surface $F^{\prime}$ with its boundary components capped by copies of $D$ is a Heegaard splitting surface $S$ for the Lens space $V \cup V^{\prime}$. Now we use exactly the same method as in [Bon] to isotope the singular curve of the spine, i.e. of $A_{0}$, onto the Heegaard surface $S$. It is easily checked that this isotopy can be done inside $V$.

We now remove simple closed curves of intersection of int $A_{0}$ and $F^{\prime}$ : The handlebodies $H$ and $H^{\prime}$ which are the closures of the complements of $V-F^{\prime}$ (after the trivial annuli components of $F \cap V$ are removed off $V$ ) have complete systems of meridian disks that are either boundary compressions of the annuli of $F^{\prime}$ or meridian disks of the tubes (Lemma 2.2). We now apply a version of Haken's argument, Lemma 8 of [BO], to push pieces of $A_{0}$ across $F^{\prime}$ using innermost sub-disks of the meridians of $H$ and $H^{\prime}$ which are disjoint from $\partial V$. With respect to these moves, which take plece in the interior of $V$, the Heegaard surface $F^{\prime}$ for $V$ behaves the same as a Heegaard surface of a closed lens space. We conclude that $A_{0} \cap F^{\prime}$ is a collection of $\operatorname{arcs} \alpha_{0}$ on $A_{0}$ with end points on $\sigma$ plus $\sigma$ itself. An innermost arc $\alpha$ in $A$ (the preimage of $\alpha_{0}$ in $A$ ) bounds a disk $D$ on $A$ (Fig. 10(a)). As in Lemma 6 of $[\mathrm{BO}]$ we can perturb the image of this disk in $V$ so it is an essential disk which intersects $\sigma$ in one point. Hence this disk is a meridian disk and $\sigma$ is a core of a handle of either $H$ or $H^{\prime}$, say $H$, see Fig. 10(b) below.

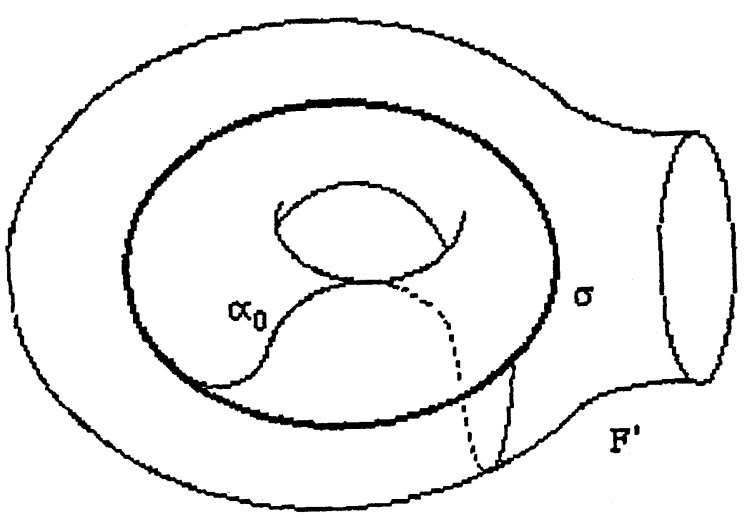

(a)

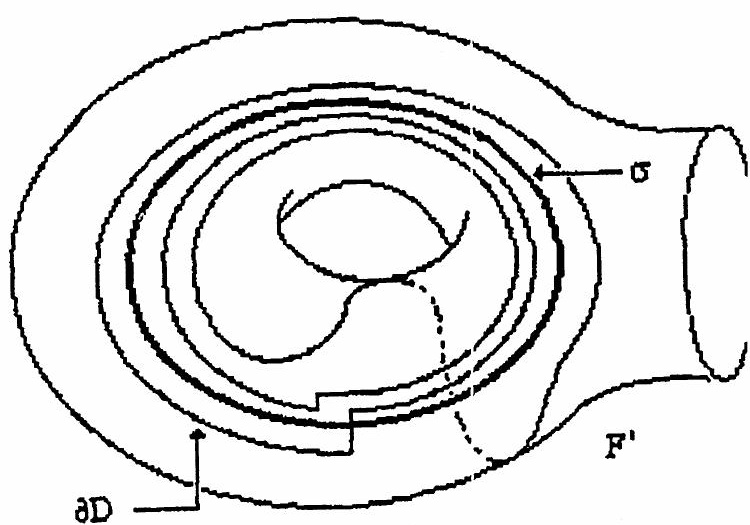

(b)

Figure 10. 
We can push $\sigma$ slightly off $F^{\prime}$ into $H$ and $A_{0} \cap F^{\prime}$ becomes a single simple closed curve. Next we define a new solid torus $\widehat{V}$ obtained by removing a small open neighborhood $N\left(A_{0}\right)$ of $A_{0}$ from $V$. Let $\widehat{F}=F^{\prime} \cap \widehat{V}$. Since $\widehat{F}$ is constructed by deleting an open annulus from $F^{\prime}, \widehat{F}$ has two more boundary components than $F^{\prime}$ does. Moreover $\widehat{F}$ splits $\widehat{V}$ into handlebodies $\widehat{H}$ and $\widehat{H}^{\prime}$. The handlebody $\widehat{H}^{\prime}$ is constructed by splitting $H^{\prime}$ along an incompressible annulus $A_{0} \cap H^{\prime}$. The handlebody $\widehat{H}$ is obtained by first removing an open neghborhood of $\sigma$, which is a core of a handle, from $H$. This gives a compression body, which is then split along an incompressible annulus in $A_{0}$ into a handlebody $\widehat{H}$.

The surface $\widehat{F}$ in $\widehat{V}$ satisfies all the conditions of Case 1 . Since a core of $V$ (i.e., $\sigma$ ) has been removed to form $\widehat{V}$, the boundary curves of $\widehat{F}$ are isotopic to a core of $\widehat{V}$. By Case 1, we conclude that $\widehat{F}$ must be either an annulus or two annuli connected by one standard tube. The first possibility cannot occur, as $\widehat{F}$ has at least four boundary curves. In the second case, it follows that $F^{\prime}$ is an annulus with a single tube attached, which is boundary parallel. This completes the proof of the proposition.

Remark 2.4. Our initial assumption that $\Sigma \cap M^{\prime}$ contains no annuli which are parallel to $\partial M^{\prime}$ is not necessary in Lemmas 2.1, 2.2 and Proposition 2.3, for such annuli can be eliminated by isotoping them into $V$, applying the arguments, and then isotoping them back into $M^{\prime}$.

\section{Finite Heegaard structures.}

Let $M, M^{\prime}, M_{q}, g_{0}$ and $\mathbb{M}$ be as in Section 0 and 1. The notation of a bumpy metric is discussed in [Wh]. It is shown there that any Riemannian metric can be approximated arbitrarily closely by such a metric. For a bumpy metric, any closed embedded minimal surface has no Jacobi fields, i.e., it is a non-degenerate critical point for the area functional. Note that the second variation for area has zero eigenvalues corresponding to Jacobi fields. The number of negative eigenvalues is called the index of instability of the minimal surface.

Theorem 3.1 [PR]. Given a closed and orientable 3-manifold with a bumpy metric and a strongly irreducible Heegaard splitting then either the Heegaard surface is isotopic to a minimal surface of index one or it is isotopic to the boundary of a regular neighborhood of a non-orientable incompressible surface with a single unknotted tube attached. 
Assume $M_{q}$ is non-Haken and has no embedded nonorientable surfaces. Perturb the metric on $M_{q}$ to be bumpy and assume that $\Sigma_{q}$ is an irreducible Heegaard surface in $M_{q}$ of genus $g$. By Theorem 3.1 of [CG] $\Sigma_{q}$ is strongly irreducible. Thus we can apply the above Theorem 3.1 and conclude that $\Sigma_{q}$ is isotopic to a minimal unstable surface denoted $\Sigma_{q}$ as well. Recall that the manifold $M^{\prime}$ which is $M$ truncated at a distance $s$ from the compact core of $M$ is embedded isometrically in $M_{q}$ for each $|q|$ large enough.

Lemma 3.2. Assume $M_{q}$ has a metric which has been perturbed to be bumpy and let $\Sigma_{q}$ be a minimal surface which is an irreducible Heegaard surface of genus $g$ in $M_{q}$. Then the area of the surfaces $\Sigma_{q}$ is uniformly bounded for all $|q|$ sufficiently large. In particular there is a real number $s$ so that if we truncate each cusp of $M$ at a distance $s$ from the compact core of $M$ to obtain the compact manifold $M^{\prime}$ then the area of $M^{\prime} \cap \Sigma_{q}$ is uniformly bounded.

Proof. Truncate $M$ at each cusp at a horotorus which is at a distance $s$ from the compact core of $M$ in the bumpy metric. In each cusp we have now a finite set of boundary slopes on the horotorus of length smaller than $2 \pi+\varepsilon$ for some $\varepsilon>0$. Hence we can do negative Dehn surgery culong all other slopes as the results of Section 1 apply to the perturbed bumpy metric. Denote the truncated manifold by $M^{\prime}$ as in Section 1. We can choose, (see Lemma 1.1) a metric on each of the manifolds $M_{q}$ in $\mathbb{M}$ (for $|q|$ large enough i.e. bigger than restrictions on each cusp). This metric restricts to an approximation of the complete hyperbolic metric of $M$ outside the horotori, and has strictly negative sectional curvatures inside the surgery sclid tori. Furthermore we can choose these metrics with sectional curvatures uniformly bounded between the constants $C^{-1}, C<0$. Denote the curvature of the surface $\Sigma_{q}$ induced by the metric $\rho_{q}$ by $K_{q}$. The Gauss-Bonnet theorem states that

$$
\int_{\Sigma_{q}} K_{q}=2 \pi(2-2 g) .
$$

If $H, \lambda_{1 q}, \lambda_{2 q}$ denote the mean and principal normal curvatures of $\Sigma_{q}$ then, as the surfaces are minimal, $H=\lambda_{1 q}+\lambda_{2 q}=0$. Also $K_{q}=K_{M_{q}}+\lambda_{1 q} \lambda_{2 q}$ where $K_{M_{q}}$ are the sectional curvatures of $M_{q}$. It follows that $K_{q}=K_{M_{q}}+\lambda_{1 q} \lambda_{2 q} \leq$ $C-\left(\lambda_{1 q}\right)^{2} \leq C<0$. Therefore

$$
2 \pi(2-2 g)=\int_{\Sigma_{q}} K_{q} \leq \int_{\Sigma_{q}} C=\left(\operatorname{Area} \Sigma_{q}\right) C
$$


so

$$
\text { Area } \Sigma_{q} \leq 2 \pi(2-2 g) / C \text { for all such } q .
$$

Set $\Sigma_{q}^{\prime}=i_{q}^{-1}\left(\Sigma_{q} \cap M_{q}\right)$ where $i_{q}$ is the isometry $M^{\prime} \rightarrow M_{q}$. In particular the area of all $\Sigma_{q}^{\prime}$ in $M^{\prime}$ is uniformly bounded.

Lemma 3.3. Assume $M^{\prime}$ (resp. $M^{\prime \prime}$ ) is obtained by truncation of $M$ at distance $s$ (resp. $s+\varepsilon$ ) from the compact core. Let $M^{\prime}$ be covered by closed convex geodesic balls $U_{1}, \ldots, U_{n}$ of radius $\varepsilon$, so that $M^{\prime \prime}$ is covered by corresponding balls $U_{1}^{\prime \prime}, \ldots, U_{n}^{\prime \prime}$ of radius $2 \varepsilon$ with the same centers. If $\Sigma$ is a compact embedded minimal surface in $M^{\prime \prime}$ with area bounded by $k$, then there is an integer $N(k)$ so that the total number of components of $\Sigma \cap U_{i}^{\prime \prime}$ which meet $U_{i}$, over all $i=1, \ldots, n$ is at most $N(k)$.

Proof. Suppose $R_{1}, \ldots, R_{m}$ are components of $\Sigma \cap U_{i}^{\prime \prime}$ which intersect $U_{i}$, for some $i, 1 \leq i \leq n$. Since the $U_{i}$ 's are a cover such components always exist. By monotonicity (see [Si]) the area of $R_{i}$, is at least $\lambda e^{\gamma \varepsilon}$, for some constants $\lambda, \gamma$, since $R_{i}$ runs between $\partial U_{i}^{\prime \prime}$ and $U_{i}$. If $R_{i}$ does not meet $\partial U_{i}^{\prime \prime}$ then $R_{i}$ would be contained entirely in $U_{i}^{\prime \prime}$ which cannot occur for a bumpy metric close to the complete hyperbolic metric. This is because metric spheres are convex in a negatively curved metric, so they cannot touch a minimal surface from their inside, by the maximum principle. But then we have $m \lambda e^{\gamma \varepsilon} \leq k$ and hence $m \leq(k / \lambda) e^{-\gamma \varepsilon}$. So we can choose $N(k)=(n k / \lambda) e^{-\gamma \varepsilon}$.

Let $\left(N_{1}^{*}, \ldots, N_{q}^{*}\right)$ be a vector of integers so that if $M_{q}$ is a manifold with $q=\left(q_{1}, \ldots, q_{d}\right), q_{j}=p_{j} / r_{j}$ and $\max \left\{\left|p_{j}\right|,\left|r_{j}\right|\right\}>N_{j}^{*}, j=1, \ldots, d$, then the requirements of Lemma 1.1, Corollary 1.4 and Lemmas 1.6, 3.2 and 3.3 are satisfied. The vector $\left(N_{1}^{\prime \prime}, \ldots, N_{d}^{\prime \prime}\right)$ determines in this way a subset $\mathbb{M}^{*}$ of $\mathbb{M}$. For our purpose we can restrict our attention to the set $\mathbb{M}^{*}$ instead of M.

Theorem 3.4. Assume that the manifolds $M_{q}$ in $\mathbb{M}^{*}$ are non Haken and contain no non orientable surfaces and let $g \geq g_{0}$ be some fixed integer. There is a subset $\mathbb{M}^{\prime \prime}$ of $\mathbb{M}^{*}$ determined, as above, by a vector $\left(N_{1}^{\prime \prime}, \ldots, N_{d}^{\prime \prime}\right)$, $N_{j}^{\prime \prime} \geq N_{j}^{*}$, such that if $M_{q}$ is any manifold in $\mathbb{M}^{\prime \prime}$ and $\Sigma_{q}$ an irreducible Heegaard splitting surface of $M_{q}$ of genus smaller or equal to $g$ then there is a finite collection of surfaces $\Sigma^{1}, \ldots, \Sigma^{r}$ in $M$ so that $\Sigma_{q}$ is isotopic to one of the $\Sigma^{i}$ 's. 
Proof. As the manifolds $M_{q}$ are non Haken and do not contain non orientable surfaces and $\Sigma_{q}$ is an irreducible Heegaard surface we can apply Theorem 3.1 of [CG] and our Theorem 3.1 and assume that $\Sigma_{q}$ is a minimal surface. By Lemma 3.3 we can apply Theorem 3.1 of [An] only to the bounded number of connnected bounded components of $\Sigma_{q} \cap U_{i}^{\prime \prime}$ which intersect $U_{i}$, for each $i=1, \ldots, n$. Note that the components of $\Sigma_{q} \cap U_{i}^{\prime \prime}$ need not have a uniformly bounded number of boundary curves, as required in Theorem 3.1 of $[\mathrm{An}]$. The condition that the number of boundary curves be bounded is used in the proof of Theorem 3.1 of [An] to show that in the limit of a convergent subsequence of such surfaces the curvature cannot blow up and handles cannot pinch at more than a finite number of points in each $U_{i}$. This is also true in our case because the genus of the surfaces $\Sigma_{q}$ is fixed, so if arbitrarily large numbers of handles do pinch in some $U_{i}$, then marly planar surface components must exist in the limit surface, all meeting $U_{i}$. As in Lemma 3.3, we get a contradiction to monotonicity, since the limit surface must have bounded area.

We conclude from Theorem 3.1 of [An] that on each $U_{i}$ there is a convergent subsequence of the surfaces $\Sigma_{q} \cap U_{i}$. As there are only finitely many $U_{i}$ 's we can piece together a subsequence $\Sigma_{q_{j}}^{\prime}$ of the surfaces $\Sigma_{q}^{\prime}$ which converges on $M^{\prime}$.

Either the surfaces $\Sigma_{q_{j}}^{\prime}$ converge smoothly to a properly embedded smoth compact minimal surface $\Sigma^{\prime}$, or they converge in the weak topology on varifolds to a properly embedded smooth compact minimal surface $\Sigma^{\prime}$ of smaller Euler characteristic with multiplicity $\geq 2$. Convergence in the weak topology means that there are a finite number of tubes in the surface which pinch at the limit and the convergence is smooth away from these points. Moreover the curvature of the surfaces $\Sigma_{q_{j}}^{\prime}$ remains bounded except in small neighborhoods of a finite collection of points on $\Sigma^{\prime}$ where the tubes are pinched.

The proof will be completed by the follwing lemmas which given the fact that the surfaces converge describe the nature of the converging process and the converging surfaces.

Lemma 3.5. Except for finitely many surfaces the sequence $\Sigma_{q_{j}}^{\prime}$ is contained in a small regular neighborhood of $\Sigma^{\prime}$.

Proof. If the convergence is smooth we are done. So suppose that the claim does not hold. Then there is a subsequence of surfaces and points on them which converge to a point $p$ a distance $d$ from $\Sigma^{\prime}$. A ball of radius $d / 2$ about $p$ intersects the surfaces in some pieces and by the monotonicity formula the area of each such piece is at least $\beta e^{\gamma d}$, for some constants $\beta, \gamma$. T.his is a 
contradiction as this subsequence converges in the weak topology to $\Sigma^{\prime}$ and hence must have almost all of its area in an arbitrarily small neighborhood of $\Sigma^{\prime}$.

Choose $s=3 g t$ where $t$ is the bound on the length of tubes from Lemma 1.6 and $g$ is the genus of the surfaces $\Sigma_{q}$.

Lemma 3.6. For each cusp there is some number $r<s=3$ tg so that if we truncate $M^{\prime}$ in that cusp at a distance $r$ from the compact core, to obtain a manifold $M^{*}$, the surfaces $\Sigma_{q_{j}}^{*}=\Sigma_{q_{j}} \cap M^{*}$ intersect $\partial M^{*}$, up to isotopy, in a collection of essential simple closed curves on $\partial M^{*}$.

Proof. Note that any collection of more than $3 g-3$ disjoint essential simple closed curves on $\Sigma_{q_{j}}$ contains parallel pairs. Consider the $m$-th cusp. Choose $3 g-1$ horotori $\mathcal{T}_{1}, \ldots, \mathcal{T}_{3 g-1}$ in the cusp, which are a distance $t$ apart and are perturbed so as to be transverse to $\Sigma^{\prime}$ and so that $\mathcal{T}_{i} \cap \Sigma^{\prime}$ has no points where the converging subsequence $\Sigma_{q_{j}}$ has curvature blowing up. Then for $j$ large enough $\Sigma_{q_{j}}$ is transverse to each $\mathcal{T}_{i}$ and $\mathcal{T}_{i} \cap \Sigma_{q_{j}}$ is a family of curves isotopic to a multiple of the set of curves $\mathcal{T}_{i} \cap \Sigma^{\prime}$ on the limit surface (as the convergence is smooth there).

If for some $i, \mathcal{T}_{i} \cap \Sigma^{\prime}$ contains only essential simple closed curves on $\mathcal{T}_{i}$, then we can truncate $M^{\prime}$ at $\mathcal{T}_{i}$ to obtain $M^{*}$. So we assume in contradiction that $\mathcal{T}_{i} \cap \Sigma^{\prime}$ and hence $\mathcal{T}_{i} \cap \Sigma_{q_{j}}$ (for $j$ large enough) contains loops which are contractible on $\mathcal{T}_{i}$, for all $i=1, \ldots, 3 g-1$. There are two cases to consider. Assume first that there are two horotori $\mathcal{T}_{u}$ and $\mathcal{T}_{v}$ so that all curves of $\mathcal{T}_{u} \cap \Sigma^{\prime}$ and also $\mathcal{T}_{v} \cap \Sigma^{\prime}$ are contractible on $\Sigma^{\prime}$. Then for $j$ sufficiently large, all the loops of $\mathcal{T}_{u} \cap \Sigma_{q_{j}}$ and $\mathcal{T}_{v} \cap \Sigma_{q_{j}}$ are contractible on $\Sigma_{q_{j}}$. Let $R$ be the region between $\mathcal{T}_{u}$ and $\mathcal{T}_{v}$. As the surface inside $R$ lifts to the universal cover of $R$ and contains a tube of length bigger than $t$, we can apply the argument of Lemma 1.6 to give a contradiction in this case. Hence there is at most one horotorus $\mathcal{T}_{u}$ for which all loops of $\mathcal{T}_{u} \cap \Sigma^{\prime}$ and $\mathcal{T}_{v} \cap \Sigma_{q_{j}}$, for $j$ large, are in essential in $\Sigma^{\prime}$ and $\Sigma_{q_{j}}$ respectively. By our observation in the first paragraph above, there must be a pair of parallel simple closed curves $C_{i}, C_{k}$ of $\mathcal{T}_{i} \cap \Sigma_{q_{j}}$ and $\mathcal{T}_{k} \cap \Sigma_{q_{j}}$, which are contractible on $\mathcal{T}_{i}$ and $\mathcal{T}_{k}$ but are essential on $\Sigma_{q_{j}}$ respectively, so that $C_{i} \cup C_{k}$ bounds an annulus $T$ on $\Sigma_{q_{j}}$. If $T$ is contained in the region $R$ between $\mathcal{T}_{i}$ and $\mathcal{T}_{k}$ then we again have a contradiction to Lemma 1.6. Moreover the argument in Lemma 1.6 shows that the surface $R \cap \Sigma_{q_{j}}$ cannot have all its boundary curves contractible on either $\mathcal{T}_{i}$ or $\mathcal{T}_{k}$. 
The only other possibility is that $T$ is not contained in $R$ and there is a simple curve $C$ on, say $\mathcal{T}_{i} \cap T$, such that $C$ is essential on $\mathcal{T}_{i}$. Clearly, either $C$ bounds a disk in $T$ or $C$ is parallel to $C_{i}$ (and $C_{k}$ ) in $T$. As $C_{i}$ is inessential in $\mathcal{T}_{i}$ it follows that $C$ is null homotopic in $M_{q}$ in both cases. Now $\mathcal{T}_{i}$ bounds a solid torus $V_{i}$ in $M_{q}$ and the only essential simple closed curve on $\mathcal{T}_{i}=\partial V_{i}$ which is null homotopic in $M_{q}$ is a meridian curve for $V_{i}$. As $j \rightarrow \infty$, the minimum length $\ell$ of this curve also goes to infinity, on the fixed horotorus $\mathcal{T}_{i}$

Assume without loss of generality that $i<k$ and consider the intersection of $T$ with the region $R^{\prime}$ between $\mathcal{T}_{i-1}$ and $\mathcal{T}_{i}$. if some transverse horotorus $\mathcal{T}$ between $\mathcal{T}_{i-1}$ and $\mathcal{T}_{i}$ meets $T$ in only inessential curves, we can enlarge $R$ to the region between $\mathcal{T}$ and $\mathcal{T}_{k}$ and get a contradiction by Lemma 1.6. If for all such horotori $\mathcal{T}, \mathcal{T} \cap T$ always contains essential (meridional.) curves, then by the coarea formula (see [Si]), the area of $T$ in $R^{\prime}$ is at least $t \ell$ and $t \ell \rightarrow \infty$ as $|q| \rightarrow \infty$ which is a contradiction to the fact that the area of all surfaces is uniformly bounded. Repeating this argument in each cusp completes the proof of Lemma 3.6.

Lemma 3.7. The points of the surface $\Sigma^{*}$ where the convergence is not smooth have neighborhoods $B_{1}, \ldots, B_{k}$ so that if $\left|q_{j}\right|$ is sufficiently large the intersection $\Sigma_{q_{j}}^{*} \cap B$, for each surface $\Sigma_{q_{j}}^{*}$, is a collection of standard, perhaps nested, annuli plus some disks.

Remark 3.8. By a standard annulus in a handlebody we mean a boundary parallel annulus. In this case it is an annulus which has an essential arc $\alpha$ that, together with $\operatorname{arc} \delta$ on $\partial B_{i}$ bounds an embedded disk in $\operatorname{cl}\left(E_{i}-\Sigma_{q_{j}}^{*}\right)$.

Proof. The surfaces $\Sigma_{q_{j}}^{*}$ converge smoothly to $\Sigma^{*}$ except for a finite collection of points of $\Sigma^{*}$. These points have small neighborhoods $B_{1}, \ldots, B_{k}$ in $M^{*}$ where the curvature of $\Sigma_{q_{j}}^{*}$ blows up and handles pinch out as $\Sigma_{q_{j}}^{*}$ converge to $\Sigma^{*}$. Assume that on $M^{*}-\left\{B_{1}, \ldots, B_{k}\right\}$ the surfaces $\Sigma_{q_{j}}^{*}$ converge to $\Sigma^{*}$ with multiplicity $m$. Hence for $j$ large enough $\Sigma_{q_{j}}^{*} \cap \partial B_{i}$ is a collection of parallel circles converging to the single circle $\Sigma^{*} \cap \partial B_{i}$.

We can now apply a similar argument as in Proposition 2.3 inside each $B_{i}$. The components of $\partial B_{i}-\Sigma_{q_{j}}^{*}$ are two disks and a collection of annuli. The annuli are incompressible in the parts of the handlebodies $H, H^{\prime \prime}$ outside $B_{1} \cup \cdots \cup B_{k}$, since otherwise a compressing disk for such an annulus being disjoint from $\Sigma_{q_{j}}^{*}$ and outside $B_{i}$ would be contained between the sheets of $\Sigma_{q_{j}}^{*}$ thus converging to $\Sigma^{*}$. Hence the sheets of $\Sigma_{q_{j}}^{*}-\left(B_{1} \cup \cdots \cup B_{k}\right)$ would be 
disks and $\Sigma_{q_{j}}^{*}$ would be a closed surface (the disks together with the parts of the surface $\Sigma_{q_{j}}^{*}$ which are in $B_{i}$ ) not meeting the horotori $\mathcal{T}$ at all.

If on the other hand an annulus of $\partial B_{i}-\Sigma_{q_{j}}^{*}$ has a compressing disk $D$ in $B_{i}$, then $B_{i}$ splits along $D$ into two 3 -balls containing components of $\Sigma_{q_{j}}^{*} \cap B_{i}$. If there is a unique compressible such annulus it, i.e. $\Sigma_{q_{j}}^{*}$, splits $B_{i}$ into handlebodies. It is readily seen by induction on the number of compressible annuli, that each of these 3-balls is split by $\Sigma_{q_{j}}^{*}$ into handlebodies and glueing back along disks $D$ gives a splitting of $B_{i}$ into handlebodies.

It is convenient to first assume that there are no annuli of $\Sigma^{*}-B_{1} \cup \ldots \cup B_{k}$ which are parallel to annuli on $\partial B_{i}$. At the end of the argument we will see that this hypothesis is unnecessary as in Section 2. The two disk components of $\partial B_{i}-\Sigma_{q_{j}}^{*}$ represent innermost tubes (after throwing away trivial disk components of $\Sigma_{q_{j}}^{*} \cap B_{i}$ ) which determine a handlebody $H$. as the annuli are contained in one or other of the handlebodies $H, H^{\prime}$ they must boundary compress there. Consider a boundary compression $D^{\prime}$ for an innermost annulus of $\partial B_{i}-\Sigma_{q_{j}}^{*}$ in $H^{\prime}$ (see Fig. 11). The disk $D^{\prime}$ must be in $B_{i}$ as otherwise strong irreducibility of the Heegaard splittings will be violated.

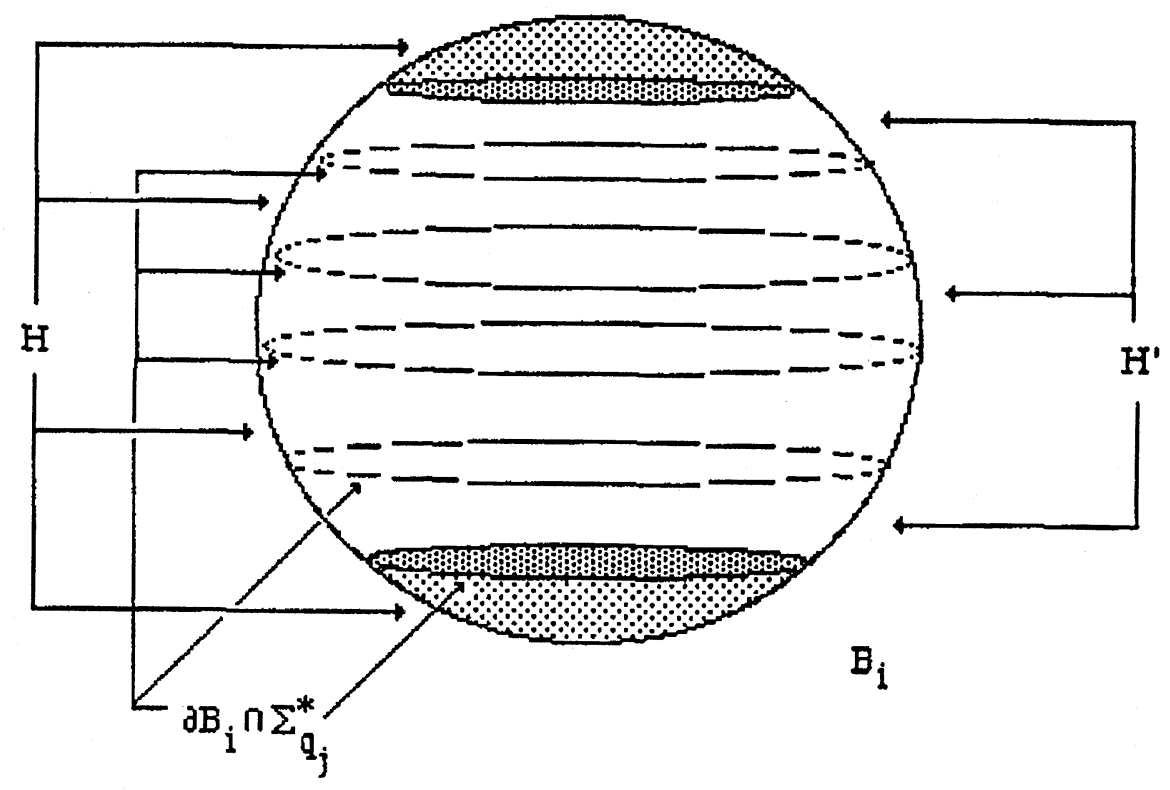

Figure 11.

After compressing the annulus along $D^{\prime}$ we see a disk in $\partial B_{i} \cap H^{\prime}$. This disk cannot be essential in $H^{\prime}$ as that would violate the strong irreducibility of 
the Heegaard splitting, as before in Section 2. Therefore the disk is parallel into $\Sigma_{q_{j}}^{*}=\partial H^{\prime}$. Hence this component of $\Sigma_{q_{j}}^{*} \cap B_{i}$ meeting $D^{\prime}$ is a standard annulus. We can now pull this annulus outside $\Sigma_{q_{j}}^{*} \cap B_{i}$ reducing the number of annuli and repeat the argument. Once $\Sigma_{q_{j}}^{*} \cap \partial B_{i}$ has only two curves left strong irreducibility is no longer violated when we compress hence we need to use Waldhausen's theorem on the uniqueness of Heegaard splittings of $S^{3}$ [Wa1] to conclude that $\Sigma_{q_{j}}^{*} \cap B_{i}$ is either two disks or a standard annulus. We conclude that $\Sigma_{q_{j}}^{*} \cap B_{i}$ is a collection of standard annuli and disks.

Remark 3.9. If, as $j \rightarrow \infty$, annuli keep appearing in $\Sigma_{q_{j}}^{*} \cap B_{i}$ then these annuli will pinch in the limit.

Lemma 3.10. If the surfaces $\Sigma_{q_{j}}^{*}$ converge to $\Sigma^{*}$ with multiplicity $m>1$ then $k$, the number of balls $B_{i}$, is one and the number of standard annuli at most $[m / 2]$.

Proof. We know by Theorem 3.1 that each $\Sigma_{q_{j}}$ is a minimal surface of index one. Also by a result of Schoen, (see [Sc]), if a minimal surface in a ball with fixed metric has principal curvature sufficiently large then it is unstable. Hence for $j$ large enough each ball contributes to the index of instability of $\Sigma_{q_{j}}$. Consequently there is at most one ball $B$ and $k=1$. Finally there are $m$ boundary curves of $\Sigma_{q_{j}}^{*} \cap B$ so by Lemma 3.8 there are at most [m/2] standard annuli.

Remark. Since $\Sigma_{q_{j}}^{*}$ is connected, it is easy to see that if $m>1$ then in fact $m$ must be 2 , in order to be able to join up the sheets of $\Sigma_{q_{j}}^{*}-B$. We do not use this fact here.

Resuming the proof of Theorem 3.4. Choose now a vector of integers $\left(N_{1}^{\prime \prime}, \ldots, N_{d}^{\prime \prime}\right), N_{j}^{\prime \prime} \geq N_{j}^{*}$ so that if $\max \left\{\left|p_{j}\right|,\left|r_{j}\right|\right\}>N_{j}, j=1, \ldots, d$ the requirements of Lemmas 3.6 and 3.7 are satisfied.

Assume now that there are infinitely many non-isotopic surfaces $\Sigma_{q}^{*}$. Then by Theorem 3.1 of [An] we have a sequence of surfaces $\Sigma_{q_{j}}^{*}$ converging to a minimal surface $\Sigma^{*}$. If the convergence is smooth, i.e., no tubes pinch, we get a contradiction as all surfaces far enough up the sub-sequence must lie inside a regular neighborhood of the limit surface and hence must be isotopic. Hence there are only finitely many surfaces $\Sigma_{q}^{\prime}$ up to diffeomorphism. So assume that some tubes pinch. Then by Lemma 3.10, the surfaces $\Sigma_{q_{j}}^{*}$ are all isotopic to at most [ $m / 2]$ copies of $\Sigma^{*}$ with a bounded number of standard tubes hence are all isotopic to a finite collection of surfaces as well. 
In order to finish the proof we need to show that there are only finitely many ways to close up the finitely many surfaces $\Sigma_{q_{j}}^{*}$, in each cusp. Consider the surfaces $\Sigma_{q}$ corresponding to $\Sigma_{q_{j}}^{*}$ for some specific $i$. If there are only finitely many indices $q$ for which $\Sigma_{q}^{*}$ is isotopic to $\Sigma_{q_{j}}^{*}$ or to two copies of $\Sigma_{q_{j}}^{*}$ with some standard tubes attached, then we disregard this particular $\Sigma_{q_{j}}^{*}$. We can assume by Lemma 3.6 that the intersection of $\Sigma_{q_{j}}^{*}$ with the level horotorus $\mathcal{T}_{r}$ is only a fixed union of essential simple closed curves. Note that as we vary the surgeries the slope of the boundary curves changes in $V$.

We can assume that any minimal surface cannot intersect any surgery torus in a disk. Hence we can apply Proposition 2.3 to conclude that the surfaces $\Sigma_{q}-\Sigma_{q}^{*}$ are a collection of annuli with at most one standard tube for each cusp. Therefore there are only finitely many ways to comlete $\Sigma_{q}^{*}$ to $\Sigma_{q}$ by adding annuli plus possibly one tube to the boundary curves in each cusp. It is clear by general position that we can isotope the tube off the core of each surgery torus. Thus the surfaces $\Sigma_{q}$ can be pushed into $M^{*} \subset M$ and there are only finitely many surfaces $\Sigma_{q}$ up to isotopy. This completes the proof of Theorem 3.4.

\section{Non orientable surfaces and Haken manifolds.}

In this section we prove a version of Theorem 3.4 for manifolds $M_{q}$ which either contain non-orientable surfaces or are Haken.

Proposition 4.1. Suppose the manifolds $M_{q}$ in $\mathbb{M}^{\prime \prime}$ are non-Haken but contain non-orientable surfaces and let $g \geq g_{0}$ be a fixed integer. Let $\Sigma_{q}$ be an irreducible Heegaard splitting surface of $M_{q}$ of genus smaller or equal to g. Then there is a finite collection of surfaces $\Sigma^{1}, \ldots, \Sigma^{r}$ in $M$ so that $\Sigma_{q}$ is isotopic to one of the $\Sigma^{i}$ 's.

Proof. If there are only finitely many manifolds $M_{q}$ which contain nonorientable surfaces, ignore them and use Theorem 3.4. This might require to increase the size of the allowed $|q|$. Hence we assume by Theorem 3.1 (c.f. [PR]) that there are infinitely many manifolds $M_{q}$ in which the Heegaard surface $\Sigma_{q}$ is isotopic to the boundary of a regular neighborhood of a nonorientable incompressible surface $S_{q}$ with a single unknotted tube attached. The surfaces $S_{q}$ define surfaces $S_{q}^{*}$ in $M^{*}$ and by [MSY] each such surface is isotopic to a least area surface $S_{q}^{*}$ in its isotopy class. Arguing just as in Theorem 3.4 these surfaces converge. As there is a standard way to reconstruct $\Sigma_{q}^{*}$ from $S_{q}^{*}$ there are only finitely classes of $\Sigma_{q}^{*}$. The proof is 
finished by an application of Proposition 2.3 to close the surfaces up. Note that in this case there is only a single tube joining the two sheets and so we do not get additional tubes in $V$. In fact $S_{q}$ cannot have any tubes in $V$ as it is incompressible.

In order to prove the theorem for Haken manifolds we need the following definition due to Schultens (see [Sh]).

Definition 4.2. Let $R$ be a closed surface contained in the boundary of a 3-manifold $M$. Let $U_{1}, U_{2}$ be a pair of compression bodies defining a Heegaard splitting for $M$, and assume that $R \subset \partial U_{1}$. Note that there is some component $R^{\prime} \subset \partial U_{1}$ ( $R^{\prime}$ can be empty) so that $U_{1}=N\left(R \cup R^{\prime}\right) \cup 1$ handles. Let $h$ be a homeomorphism $h: N(R) \rightarrow R \times I$ and $p: R \times I \rightarrow R$ the projection onto the first factor.

Let $M_{1}, M_{2}$ be two manifolds each with non-empty boundary and with Heegaard splittings $\left(U_{1}, U_{2}\right),\left(V_{1}, V_{2}\right)$ respectively. Let $R_{1}, R_{2}$ be two homeomorphic surfaces such that $R_{1} \subset \partial U_{1} \subset \partial M_{1}$ and $R_{2} \subset \partial V_{1} \subset \partial M_{2}$ and let $h_{i}, p_{i}, i=1,2$, be the corresponding functions respectively.

Define an equivalence relation $\sim$ on $M_{1} \cup M_{2}$ as follows:

1) If $x_{i}, y_{i}$ are points such that $x_{i}, y_{i} \in N\left(R_{i}\right)$ and $p_{i} h_{i}\left(x_{i}\right)=p_{i} h_{i}\left(y_{i}\right)$ then $x_{i} \sim y_{i}$.

2) If $x \in R_{1}, y \in R_{2}$ and $g(x)=y$, where $g: R_{1} \rightarrow R_{2}$ is the homeomorphism between the surfaces, then $x \sim y$.

Futhermore we can arrange that the attaching disks on $R_{1} \times I\left(R_{2} \times I\right)$ for the one handles in $U_{1}\left(V_{1}\right)$ respectively, have disjoint images in $R_{1}\left(R_{2}\right)$ and hence they do not get identified to each other. Now set:

$$
M=\left(M_{1} \cup M_{2}\right) / \sim, \quad W_{1}=\left(U_{1} \cup V_{2}\right) / \sim, \quad W_{2}=\left(U_{2} \cup V_{1 .}\right) / \sim .
$$

Note that $W_{1}=V_{2} \cup N\left(R_{1}^{\prime}\right) \cup$ (1-handles) and $W_{2}=U_{2} \cup N\left(R_{2}^{\prime}\right) \cup$ (1-handles) (the 1-handles connect $\partial_{+} V_{2}$ to $\partial N\left(R_{1}^{\prime}\right)\left(\partial_{+} U_{2}\right.$ to $\partial N\left(R_{2}^{\prime}\right)$ respectively)) so that $W_{1}, W_{2}$ are compression bodies defining a Heegaard splitting $\left(W_{1}, W_{2}\right)$ for $M$.

The Heegaard splitting $\left(W_{1}, W_{2}\right)$ of $M$ is called the amalgamation of the Heegaard splittings $\left(U_{1}, U_{2}\right)$ of $M_{1}$ and $\left(V_{1}, V_{2}\right)$ of $M_{2}$ along $R_{1}, R_{2}$ via the homeomorphism $g$.

Theorem 4.3. Suppose the manifolds $M_{q}$ in $M^{\prime \prime}$ are Haken or contain non-orientable surfaces and let $g \geq g_{0}$ be a fixed integer. There is a subset 
$\mathbb{M}^{0}$ of $\mathbb{M}^{\prime \prime}$ determined, as above, by a vector $\left(N_{1}^{\prime}, \ldots, N_{d}^{\prime}\right) N_{j}^{\prime} \geq N_{j}^{\prime \prime}$, such that if $M_{q}$ is any manifold in $\mathbb{M}^{0}$ and $\Sigma_{q}$ is an irreducible genus $g$ Heegaard splitting surface of $M_{q}$ for $g \geq g_{0}$, then there is a finite collection of surfaces $\Sigma^{1}, \ldots, \Sigma^{r}$ in $M$ so that $\Sigma_{q}$ is isotopic to one of the $\Sigma^{i}$ 's.

Proof. In the case that $\Sigma_{q}$ is strongly irreducible regardless of whether it is Haken or contains non-orientable surfaces, we can apply Theorem 3.4 and Proposition 4.1 to obtain the claim. This is because the only place that we used the fact that $M_{q}$ is non-Haken in the proof of Theorem 3.4 is to conclude that $\Sigma_{q}$ is strongly irreducible.

If $\Sigma_{q}$ is weakly reducible (i.e. not strongly irreducible) there is a maximal system of disks $\Delta$ in each $M_{q}$ so that after compressing $\Sigma_{q}$ along $\Delta$ and throwing away any trivial 2-sphere components we obtain a closed incompressible surface $L$ in $M_{q}$. As $M_{q}$ has a negatively curved metric, $L$ cannot have any essential 2-sphere or torus components. The surface $L$ can be isotoped to a least area surface, again denoted by $L$ (by [MSY]). Since genus $(L) \leq \operatorname{genus}\left(\Sigma_{q}\right)-2=g-2$, then by Lemma 3.2 Area $(L) \leq 2 \pi(2-2(g-2)) / C$. Hence as in Corollary 1.4, for $|q|$ large enough, $L$ cannot intersect any of surgery solid tori $V$, attached at the cusp, in meridian disks. Since $L$ is minimal and has bounded area we can choose a horotorus $\mathcal{T}_{r}$ for each cusp of $M$, as in Theorem 3.6 so that $\mathcal{T}_{r} \cap L$ is composed only of essential curves. Therefore, since $L$ is incompressible, it follows that at this cusp $V \cap L$ must consist entirely of annuli. Now it is easy to check that in a negatively curved solid torus with pinched curvature close to -1 , a least area annulus must be very close to the boundary. (For example, use the coarea formula, (see [Si]) to compare a "long" annulus with one near the boundary.) So we can find a smaller solid torus inside $V$ which is disjoint from $L$. Hence $L$ is contained in $M^{*}$ which is $M^{\prime}$ with cusps truncated at a suitable choice of horotori $\mathcal{T}$. Notice that by Haken's finiteness theorem (see [Ha]), since $M^{*}$ is negatively curved and hence irreducible and atoroidal and genus $(L) \leq g-2$ is bounded, there are only finitely many isotopy classes for such incompressible surfaces $L$ in $M^{*}$.

Since $L$ is homologous to $\Sigma_{q}$ in $M_{q}$ it follows that $L$ separates $M_{q}$. We can split $M_{q}$ along $L$ into several components $M_{q}^{1}, \ldots, M_{q}^{n}$ and there are only finitely many possibilities to do so. Since $L$ is contained in $M^{*}$ the surgery solid tori on cusps of $M$ are contained in the components $M_{q}^{1}, \ldots, M_{q}^{n}$. The Heegaard surface $\Sigma_{q}$ of $M_{q}$ induces Heegaard splittings on the components $M_{q}^{1}, \ldots, M_{q}^{n}$ as follows: 
Let $\left(H_{1}, H_{2}\right)$ be the handlebodies of $M$ with splitting surface $\Sigma_{q}$. As $\Sigma_{q}$ is weakly reducible let $\Delta$ be a maximal system of disjoint non parallel compressing disks. Consider $M_{q}^{i}$ the closure of a component of $M-L$. We can assume that $M_{q}^{i} \subset H_{1} \cup N\left(\Delta_{2}\right)$, where $\Delta=\Delta_{1} \cup \Delta_{2}$ and $\Delta_{i}$ is the sub-collection of $\Delta$ consisting of compression disks for $\Sigma_{q}$ in $H_{i}$. Set $U_{1}=H_{1} \cap M_{q}^{i}$. We can obtain $M_{q}^{i}$ from $U_{1}$ by attaching 2-handles and hence one can obtain $U_{1}$ from $M_{q}^{i}$ by removing 2-handles (i.e., by drilling out tunnels), thus $U_{1}$ is connected. So $U_{1}$ is a single component of $H_{: l}-N\left(\Delta_{1}\right)$ and hence is a compression body. Now $U_{2}=M_{q}^{i}-U_{1}$ is obtained from a collar of $M_{q}^{i} \cap \Sigma^{*}$ by attaching 1-handles. It is connected because $\partial_{+} U_{1}=\partial_{+} U_{2}$ and therefore it is also a compression body. Thus $\left(U_{1}^{i}, U_{2}^{i}\right)$ is a Heegaard splitting for $M_{q}^{i}$. It is called the induced Heegaard splitting on $M_{q}^{i}$. Denote the induced Heegaard surface $\partial U_{1}^{i}=\partial U_{2}^{i}$ by $\Sigma_{q}^{i}$. Note that the component of $L$ which is $M_{q}^{i}$ is contained either entirely in $\partial_{-} U_{1}$ or entirely in $\partial_{-} U_{2}$.

Conversely, assume that the components $M_{q}^{1}, \ldots, M_{q}^{n}$ have Heegaard splittings $\Sigma_{q}^{i}$. We glue the components $M_{q}^{1}, \ldots, M_{q}^{n}$ together along the components of $L$ to obtain $M_{q}$. Then since regular neighborhoods are unique up to isotopy, we can amalgamate the Heegaard splittings $\Sigma_{q}^{i}$ via the identity map on the components of $L$ to a well defined Heegaard splitting $\Sigma_{q}$ of $M_{q}$. Each $\Sigma_{q}^{i}$ has genus less than $g$, but the total genus of all the surfaces $\Sigma_{q}^{i}$ is at most $g+(g-2)=2 g-2$, since an "extra copy" of all the components of $L$ is used in this process.

As $L$ is a minimal surface, each component of $L$ forms a barrier needed for applying the minimax method of [PR] so we can apply Theorem 3.1. So if $\Sigma_{q}^{i}$ is strongly irreducible, either it is isotopic to an unstable minimal surface in $M_{q}^{i}$ or it is isotopic to the boundary of a regular neighborhood of an incompressible non-orientable surface with a single unknotted tube attached. In either case, we can use Theorem 3.4 or Proposition 4.1 to show that the number of isotopy classes of $\Sigma_{q}^{i}$, for $|q|$ sufficiently large in each of components $M_{q}^{i}$ is finite: This is because Theorem 3.1 of [An] is also true in the case of 3-manifold with boundary which is a minimal surface (which is the case here as we have arranged $L$ to be minimal).

Now choose integers $N_{1}^{\prime}, \ldots, N_{d}^{\prime}$ determining a sub set $\mathbb{M}^{0}$ of $\mathbb{M}$ so that if $q_{j}=p_{j} / r_{j}$ and $\max \left\{\left|p_{j}\right|,\left|r_{j}\right|\right\}>N_{j}^{\prime}, j=1, \ldots, d$, then the requirements of Theorem 3.4 and Proposition 4.1 hold for each of the ccmponents $M_{q}^{1}, \ldots, M_{q}^{n}$. As there are finitely many surfaces in each of the components there are finitely many amalgamated surfaces $\Sigma_{q}$ in $M_{q}$ up tp isotopy. By Proposition 2.8 of [Sh] the original Heegaard surface $\Sigma_{q}$ is isotopic to one of the finitely many amalgamated surfaces. 
Finally, if $\Sigma_{q}^{i}$ is not strongly irreducible, then it can be compressed further using disjoint disks in both its compression bodies, to give a new incompressible surface $L^{\prime}$ in $M_{q}^{i}$ which is not boundary parallel. We split $M_{q}^{i}$ along $L^{\prime}$ and apply the argument again to get a new Heegaard splitting for the components of $M_{q}^{i}-L$. Again by Haken's finiteness results (see [Ha]), this process can occur only a bounded number of times, so that eventually we must get strongly irreducible splittings. That is $M_{q}$ is split along a maximal system of incompressible surfaces coming from $\Sigma_{q}$. Note that we need to adjust the vector $N_{1}^{\prime}, \ldots, N_{d}^{\prime}$ of integers and the set $\mathbb{M}^{0}$ each time we apply the above argument. This conpletes the proof of Theorem 4.3.

\section{Proof of Theorem 0.1.}

Proof of Theorem 0.1. Choose a $d$-tuple of integers $\left(N_{1}, \ldots, N_{d}\right)$ so that for all $|q|$ bigger than $\left|q^{0}\right|=\min \left\{\left|q_{1}\right|, \ldots,\left|q_{d}\right|\right\}$ where $q_{j}=p_{j} / r_{j}$ and $\max \left\{\left|p_{j}\right|,\left|r_{j}\right|\right\}>N_{j}, j=1, \ldots, d$ the requirements of Theorems 3.4, 4.3 and Proposition 4.3 are satisfied and let $g \geq g_{0}$ be some positive integer. Then it follows that any irreducible Heegaard surface $\Sigma_{q}$ of genus $\leq g$ of $M_{q}$ is isotopic to some surface $\Sigma^{i}$ in a finite collection $\left\{\Sigma^{1}, \ldots, \Sigma^{r}\right\}$. Denote this surface also by $\Sigma_{q}$. It follows from the proof of Theorem 3.4 that for each cusp in any of the manifolds in $\mathbb{M}^{\prime}$ we can isotope the surface $\Sigma_{q}$ off the core of the surgery torus. Furthermore this isotopy can be taken to be the identity map outside a small torus neighborhood of the core.

Consider a handlebody $H$ bounded by $\Sigma_{q}$, and assume it contains geodesics, corresponding to a subset $\left\{c_{i_{1}}, \ldots, c_{i_{t}}\right\}$ of the cusps, forming a link $\mathcal{L} \subset H$. Compress $\Sigma_{q}$ as much as possible in the complement of $\mathcal{L}$. Let $\Sigma_{q}^{\prime}$ be a component of the resulting surface, (possibly disconnected), bounding a handlebody $H^{\prime}$ (after surgery) containing a sublink $\mathcal{L}^{\prime}$ of $\mathcal{L}$ so that $\Sigma_{q}^{\prime}$ is incompressible in $H^{\prime}-\mathcal{L}^{\prime}$. As $H^{\prime}-\mathcal{L}^{\prime}$ is a Haken manifold it has a unique torus decomposition into manifolds whose interiors admit geometric structures (see [Th2]).

We consider the various possibilities.

a) Assume $H^{\prime}-\mathcal{L}^{\prime}$ contains no incompressible annuli. If $H^{\prime}-\mathcal{L}^{\prime}$ does not contain nonboundary parallel incompressible tori then it is hyperbolic. By doing negatively curved Dehn surgery on the cusps $\left\{c_{i_{1}}, \ldots, c_{i_{t}}\right\}$ we obtain a handlebody with a complete metric of negative curvature and finite volume. The boundary is a totally geodesic surface in a handlebody so it must compress and this is a contradiction. If $H^{\prime}-\mathcal{L}^{\prime}$ does contain 
non-boundary parallel incompressible tori these tori must compress after appropriate surgery on $\mathcal{L}^{\prime}$. This is because all these Dehn surgeries yield handlebodies which do not contain incompressible tori. Splitting $H^{\prime}-\mathcal{L}^{\prime}$ along these tori we obtain manifolds with no non-peripheral incompressible tori. Hence by Thurston [Th2] we have manifolds which admit a, complete hyperbolic metric. Choose an innermost disk $D^{\prime}$ on any of the compressing disks $D$ for the tori. The disk $D^{\prime}$ must be contained in a hyperbolic component $N$ of the manifold which also contains one of the original cusps and it bounds a non-trivial curve on one of the tori, say $\mathcal{T}_{0}$. This would imply that we have a compressible simple closed curve on a horotorus in the cusp of the hyperbolic manifold $N$ determined by $\mathcal{T}_{0}$, which is a contradiction.

b) Assume $H^{\prime}-\mathcal{L}^{\prime}$ contains incompressible non-boundary parallel annuli. Hence it contains Seifert fibered pieces, (see [JS]). Suppose a component of the Seifert characteristic variety contains more than one component of the link $\mathcal{L}^{\prime}$. Then either there is an incompressible annulus between two cusps which is embedded in $M$, contradicting the fact that $M$ is hyperbolic, or if this annulus is compressible in $M$ then a cusp bounds a disk in $M$, which is also impossible.

If a bounded component of the Seifert characteristic variety does not contain any component of $\mathcal{L}^{\prime}$ it is homeomorphic to an annulus $\times I$, since it is contained in $H^{\prime}$. In this case cut $H^{\prime}-\mathcal{L}^{\prime}$ along outermost such annuli repeatedly and all incompressible non-boundary parallel tori. Some component is a manifold which, after appropriate surgery on the components of $\mathcal{L}^{\prime}$, admits a hyperbolic metric and totally geodesic compressible boundary and we have the same contradiction as in (a). The boundary of the cut up manifold is compressible regardless of whether the annulus in $H^{\prime}-\mathcal{L}^{\prime}$ is compressible or not in $H^{\prime}$. Note that if the annulus $\times I$ is incompressible in $H^{\prime}$ then the cut up manifold after surgery is actually a handlebody. We conclude that a component of the relative characteristic variety of $H^{\prime}-\mathcal{L}^{\prime}$ contains only one cusp. Now there are two possible cases:

1) The relative characteristic variety of $H^{\prime}-\mathcal{L}^{\prime}$ meets the surface $\partial H^{\prime}$ in a closed surface. As the boundaries of the characteristic variety are tori or annuli this surface must be a torus. Hence $H^{\prime}-\mathcal{L}^{\prime}$ is homeomorphic to a cable space, i.e., a solid torus $D^{2} \times S^{1}$ from which a regular neighborhood of a $p, q$ torus knot on a smaller concentric torus was removed. As infinitely many surgeries $q_{i}=m_{i} / n_{i}$ where $m_{i} \neq n_{i} p q \pm 1$ on this space give a solid torus the $p, q$-cable space must be trivial, that is a 1,0-cable (see [Go], Lemma 7.2). In other words $\mathcal{L}^{\prime}$ is a core of $H^{\prime}$ and hence of $H$.

2) The characteristic variety meets the surface $\partial H^{\prime}$ in annuli. Hence there is an annulus between $\partial H^{\prime}$ and $\partial N\left(\mathcal{L}^{\prime}\right)$ (take an arc in the base orbifold 
between the corresponding boundary components and cross it with $S^{1}$ ). The curve on $\partial N\left(\mathcal{L}^{\prime}\right)$ is uniquely determined by the Seifert fibration. Now repeat the argument for each component of the compressed handlebodies of the original surface which contains a component of the link $\mathcal{L}$. If they are all cores then we are done, if not then we have at least one annulus as claimed.

\section{Super additive tunnel number knots exist.}

Proof of Theorem 0.6. The manifold $K(m / n)$ has a genus $r$ Heegaard splitting which can be seen as follows: Embed an equatorial $S^{2}$ in the pair $\left(S^{3}, K\right)$ as indicated in Fig. 12. It separates the pair $\left(S^{3}, K\right)$ into two 3balls each with a system of $r$ unknotted arcs. Consider the 3-ball $B$ on the "inside" of $S^{2}$. After removing a regular neighborhood of the knot we obtain a ball minus regular neighborhoods of the unknotted arcs, i.e., a genus $r$ handlebody. The complement is composed of the surgery solid torus together with the regular neighborhood of $r-1$ tunnels (i.e., 1-handles) and hence it is a genus $r$ handlebody. In order to prove the Theorem we need to show that $K^{\prime}(m / n)$, except for a simple (see section 1) subset of surgeries $\{m / n\}$, do not have Heegaard splittings of smaller genus.

The knot space $S^{3}-N\left(K^{\prime}\right)$ is a hyperbolic manifold, (see [Ka]), with one cusp hence we can apply. Theorem 0.1. Assume to the contrary that there is a set of surgery coefficients $\left\{m^{\prime} / n^{\prime}\right\}$ which is not simple so that $K^{\prime}\left(m^{\prime} / n^{\prime}\right)$ are all of smaller genus than $r$. For each such manifold $K^{\prime}\left(m^{\prime} / n^{\prime}\right)$ consider such a Heegaard splitting surface $\Sigma\left(m^{\prime} / n^{\prime}\right)$. By Theorem 0.1 all these surfaces are isotopic to surfaces in some finite collection. Hence there is a fixed surface $\Sigma$ in $S^{3}-N\left(K^{\prime}\right)$ and some non simple subset of surgery coefficients $\left\{m^{\prime \prime} / n^{\prime \prime}\right\}$ so that the manifolds $K\left(m^{\prime \prime} / n^{\prime \prime}\right)$ all have a Heegaard splitting isotopic to $\Sigma$. Furthermore either there is an annulus between a unique simple closed curve $\beta$ on $\partial N\left(K^{\prime}\right)$ and $\Sigma$ or $K^{\prime}$ is a core for one of the handlebodies bounded by $\Sigma$. In the first case, by Theorem 0.1 case (b) and Remark 0.2 , there is a basis $\alpha, \beta$ for the homology of $\partial N\left(K^{\prime}\right)$ so that the surgery must have been along curves $\gamma$ of the form $\gamma=\alpha+n \beta$, where $n \in \mathbb{Z}$. As the set of surgery coefficients was not simple we conclude that $K^{\prime}$ is a core of a handlebody of genus smaller than $r$ bounded by $\Sigma$. But this means that the tunnel number of $K^{\prime}$ is smaller than $r$. In particular the cardinality of a minimal generating system of $\pi_{1}\left(S^{3}-N\left(K^{\prime}\right)\right)$ is smaller than $r$. This is a contradiction since Lemma 2 of $[\mathrm{BLM}]$ it was proved that the cardinality 
of a minimal generating system of $\pi_{1}\left(S^{3}-N\left(K^{\prime}\right)\right)$ is $r$.

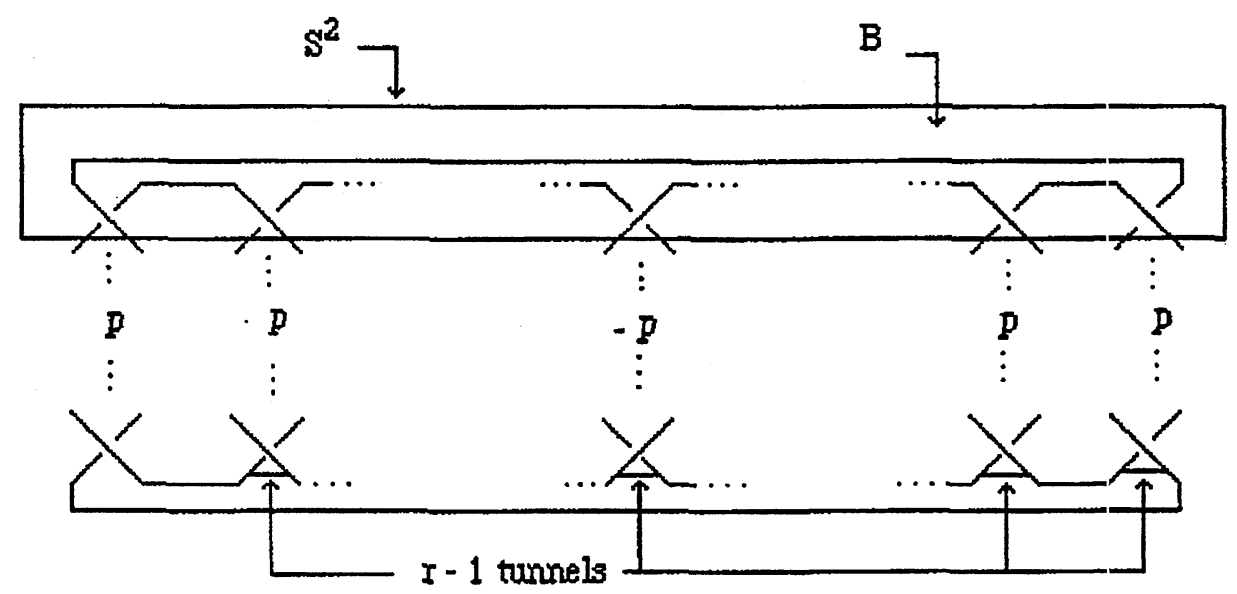

Figure 12.

Proof of Corollary 0.7. We use the proof of Theorem 1 of [BLM]. By Theorem 0.6 there exist infinitely many knots $K_{1}$ and $K_{2}$ in $S^{3}$ which are the branch sets of 2 -fold branched covers $M_{K_{1}}$ and $M_{K_{2}}$ of $S^{3}$ so that $M_{K_{1}}$ and $M_{K_{2}}$ are obtained by some surgery on the pretzel knots $K^{\prime}\left(p_{1}, \ldots, p_{r}\right)$, $K^{\prime}\left(p_{1}^{\prime}, \ldots, p_{s}^{\prime}\right)$ respectively.

In Lemma 1 of $[\mathrm{BLM}]$ it was proved that $t\left(K_{1}\right) \leq(r-1) / 2$ and $t\left(K_{2}\right) \leq$ $(s-1) / 2$. Assume also that $t\left(K_{1} \# K_{2}\right) \leq t\left(K_{1}\right)+t\left(K_{2}\right)$. This implies that $S^{3}-N\left(K_{1} \# K_{2}\right)$ has a decomposition into a handlebody $H_{1}$ of genus at most $(r+s) / 2$ and (genus $\left.H_{1}-1\right)$ 2-handles. The regular neighborhood of the tunnels and the knot is the complementary handlebody $H_{2}$. The pair $H_{1}, H_{2}$ is a Heegaard splitting of $S^{3}$ of genus $\leq(r+s) / 2$. Let $\widehat{M}$ be the 2 -fold branched cover of $S^{3}$ branched over $K_{1} \# K_{2}$. The above Heegaard splitting of $S^{3}$ lifts to a Heegaard splitting of $\widehat{M}$ of genus $\leq r+s-1$. However the 2 -fold branched cover of $S^{3}$ branched over $K_{1} \# K_{2}$ is equal to the connected sum of the two 2 -fold branched covers of $S^{3}$ branched over $K_{1}$ and $K_{2}$, i.e., $\widehat{M}=M_{K_{1}} \# M_{K_{2}}$. By Theorem 0.6 and the additivity of genus, the genus of $M^{\prime}$ is $r+s$; hence a contradiction. As the tunnel number can go up at most by one it follows that:

$$
t\left(K_{1} \# K_{2}\right)=t\left(K_{1}\right)+t\left(K_{2}\right)+1=(r+s) / 2 .
$$




\section{Manifolds $M_{q}$ of genus two.}

If the manifolds $M_{q}$ in $\mathbb{M}^{\prime}$ all have Heegaard splittings of genus two, parts of the argument simplify considerably. We first notice that the genus two Heegaard splittings are irreducible as the manifolds $M_{q}$ in $\mathbb{M}^{\prime}$ are hyperbolic. Furthermore any Heegaard splitting of genus two of $M_{q}$ is strongly irreducible. This is because by the proof of Theorem 3.1 of [CG] if the Heegaard splitting is not strongly irreducible then $M_{q}$ is Haken and we can compress the Heegaard surface to an incompressible surface $S$. An Euler characteristic argument shows that the surface $S$ must be either a torus or a 2-sphere, but hyperbolic manifolds do not contain such incompressible surfaces. Hence we can apply Theorem 3.1. In order to prove Theorem 3.4 we made a crucial use of Proposition 2.3. However in the case of genus two we have a simpler statement and a much simpler proof for this Lemma given as follows:

We use the notation of Theorem 3.4.

Lemma 7.1. Assume that the genus of the surfaces $\Sigma_{q}$ is two. Then the surfaces $\Sigma_{q}-\Sigma_{q}^{*}$ are collections of annuli.

Proof. First note that $\partial \Sigma_{q}^{*}$ is a collection of essential curves on $\mathcal{T}_{s}$ which are also essential curves in $\Sigma_{q}$ and $M_{q}$ as they are nontrivial multiples of geodesics. The surfaces $\Sigma_{q}-\Sigma_{q}^{*}$ are a collection of annuli connected by tubes. Hence the largest Euler characteristic possible of any such surface is -2 . This occurs when two annuli are connected by one tube or as one annulus with one tube attached. One option is that $\Sigma_{q}^{*}$ is two or one annuli. Each of the annuli $\Sigma_{q}^{*}$ together with the annuli on $\mathcal{T}_{s}$, which they bound, form a torus. This torus must be compressible as the manifold $M_{q}$ is hyperbolic and cannot contain incompressible tori. As $\partial \Sigma_{q}^{*}$ is a collection of essential curves on $\mathcal{T}_{s}$ and the torus is compressible, the annuli $\Sigma_{q}^{*}$ must boundary compress. Hence, since $M_{q}$ is irreducible, the annuli $\Sigma_{q}^{*}$ are boundary parallel. This implies that $\Sigma_{q}$ is isotopic into a neighborhood of a geodesic, which contradicts $\Sigma_{q}$ being a Heegaard splitting. Otherwise this surface must connect to a surface with four boundary components. The connected surface with the largest Euler characteristic and four boundary components is a four times punctured sphere. Hence the connected surface $\Sigma_{q}$ has genus three, which is a contradiction. 


\section{Remarks.}

1) J.Pitts and $H$. Rubinstein have proved in [PR] that all closed orientable 3-manifold with negative sectional curvature, have only finitely many irreducible Heegaard splittings of a given genus, up to isotopy. This proves a conjecture of Waldhausen in the negative curvature case.

2) Note that J. Hass proved in [Hs] that genus two manifolds have finitely many Heegaard splittings. This also follows from Theorem 0.1 for the manifolds in $\mathbb{M}^{\prime}$.

3) For the special case of manifolds with one cusp we can use a theorem of Ying-Qing Wu, (see [Wu]), to prove statement (b) of Theorem 0.1 and Remark 0.2 .

\section{References.}

[An] M. Andersen, Curvature estimates for minimal surfaces in 3-manifolds, Ann. Sci. Ec. Norm. Sup., (1985), 89-105.

[BH] S. Bleiler and C. Hodgson, Spherical space forms and Diehn filliings, preprint.

[BLM] M. Boileau, M. Lustig and Y. Moriah, Links with super adiditive tunnel number, to appear in J. of Camb. Phil. Soc.

[Bon] F. Bonahon, Diffeotopie des espaces lenticulaires, Topology, 22 (1983), 305-314.

[BO] F. Bonahon and J.P. Otal, Scidements de Heegaard des espaces lenticulaires, Ann. Sci. de l'ecole Norm. Sup., Vol. 16 (1983), 451-4.67.

[CG] A. Casson and C. Gordon, Reducing Heegaard splittings, Topology and its applications, 27 (1987), 275-283.

[Go] C. Gordon, Dehn surgery and satellites knots, Trans. A.M.S., Vol. 275, No. 2 (1983), 687-708.

[GT] M. Gromov and W. Thurston, Pinching constants for hyperbolic manifolds, Invent. Math., 89 (1987), 1-12.

[Ha] W. Haken, Some results on surfaces in 3-manifolds, in "Studies in Modern Topology", pp. 34-98, Math. Assoc. of America, Prentice Hall, Englewood Cliffs, NJ, 1968.

[Hs] J. Hass, Genus two Heegaard splittings, Proc. Amer. Math. Soc., Vol. 114 (1992), 565-570. 
[HS] J. Hass and P. Scott, The existence of least area surfaces in 3-manifolds, Trans. of Amer. Math. Soc., Vol. 310 (1988), 87-114.

[JS] W. Jaso and P. Shalen, Seifert fibered spaces in 3-manifolds, Mem. Amer. Math. Soc., 21 (1979).

[Ka] A. Kawauchi, Classification of pretzel knots, Kobe J. Math., 2 (1985), $1-22$.

[Mo] J. Montesinos, Surgery on links and double branched covering of $S$, knots, groups and 3-manifolds, Neuwirth L.P. (ed.), Ann. of Math. Studies, 84 (1975), 227-260, Princeton University Press.

[MS] Y. Moriah and J. Schultens, Irreducible Heegaard splittings of Seifert fibered spaces are either vertical or horizontal, preprint.

[MSY1] W. Meeks, L. Simon and T.S. Yau, Embedded minimal surfaces, exotic spheres and manifolds with positive Ricci curvature, Ann. of Math., 116(2) (1982), 621-659.

[MSY2] K. Morimoto, M. Sakuma and Y. Yokota, Concrete examples of tunnel number one knots which have that property " $1+1=3$ ", preprint.

[PR] J. Pitts and H. Rubinstein, Minimal surfaces and finiteness of Heegaard splittings of 3-manifolds.

[Si] L. Simon, Lectures on geometric measure theory, Proc. of the Center for Math. Analysis, ANU Canbarra, 1983.

[Sc] R. Schoen, Estimates for stable minimal surfaces in three dimensional manifolds, Ann. of Math. Studies, Vol. 103, Princeton University Press, 1983.

[Sh] J. Schultens, The classification of Heegaard splittings for (closed orientable surfaces) $\times S^{1}$, Proc. London Math. Soc., 67(15) (1993), 401-487.

[ST] M. Scharlemann and A. Thompson, Heegaard splittings of (surface) $\times I$ are standard, Math. Ann., 295 (1993), 549-564.

[Sw] G. Swarup, Decompositions of free groups, J. of Pure and App. Alg., 40 (1986), 99-102.

[Th1] W. Thurston, Three dimensional manifolds, Kleinian groups and hyperbolic geometry, Bull. Amer. Math. Soc., Vol. 16 (1982), 357-381.

[Th2] W. Thurston, The geometry and topology of 3-manifolds, Princeton Univ. Lecture Notes, 1978. 
[Wa1] F. Waldhausen, Heegaard-Zerlegungen der 3-sphare, Topology, 7 (1968), 195-203.

[Wa2] F. Waldhausen, On some recent results in 3-dimensional topology, Proc. Sympos. Pure Math., 64 (1977), 21-38.

[Wh] B. White, The space of minimal submanifolds of varying Riemunnian metrics, Indiana University Math. J., Vol. 40 (1991), 161-199.

[Wu] Y.Q. Wu, Incompressibility of surfaces in surgered 3-manifolds, Topology, Vol. 31 (1992), 271-279.

RECEIVED JUNE 7, 1995.

DEPARTAMENT OF MATHEMATICS

TECHNION

HAIFA 32000

ISRAEL

AND

DEPARTAMENT OF MATHEMATICS

UNIVERSITY OF MELBOURNE

PARKVILLE

VICTORIA 3057

Australia

RUBIN@MATHS.MU.OZ.AU 